\title{
Reanalysis of the binary neutron star mergers GW170817 and GW190425 using numerical-relativity calibrated waveform models
}

\author{
Tatsuya Narikawa, ${ }^{1,2, *}$ Nami Uchikata, ${ }^{2,3, \dagger}$ Kyohei Kawaguchi, ${ }^{2,4, \dagger}$ Kenta Kiuchi $\odot,{ }^{4,5, \S}$ Koutarou Kyutoku $\odot,{ }^{1,5,6,7, \|}$ \\ Masaru Shibata, ${ }^{4,5, \pi}$ and Hideyuki Tagoshi ${ }^{2, * *}$ \\ ${ }^{1}$ Department of Physics, Kyoto University, Kyoto 606-8502, Japan \\ ${ }^{2}$ Institute for Cosmic Ray Research, The University of Tokyo, Chiba 277-8582, Japan \\ ${ }^{3}$ Graduate School of Science and Technology, Niigata University, Niigata 950-2181, Japan \\ ${ }^{4}$ Max Planck Institute for Gravitational Physics (Albert Einstein Institute), Am Mühlenberg 1, Potsdam-Golm 14476, Germany \\ ${ }^{5}$ Center for Gravitational Physics, Yukawa Institute for Theoretical Physics, Kyoto University, Kyoto 606-8502, Japan \\ ${ }^{6}$ Theory Center, Institute of Particle and Nuclear Studies, KEK, Tsukuba 305-0801, Japan \\ ${ }^{7}$ Interdisciplinary Theoretical and Mathematical Science (iTHEMS) Research Group, RIKEN, Wako, Saitama 351-0198, Japan
}

(Received 31 October 2019; revised 18 June 2020; accepted 25 August 2020; published 7 October 2020)

\begin{abstract}
We reanalyze gravitational waves from binary-neutron-star mergers GW170817 and GW190425 using a numerical-relativity (NR) calibrated waveform model, the TF2+_KyotoTidal model, which includes nonlinear tidal terms. For GW170817, by imposing a uniform prior on the binary tidal deformability $\tilde{\Lambda}$, the symmetric $90 \%$ credible interval of $\tilde{\Lambda}$ is estimated to be $481_{-359}^{+436}$ and $402_{-279}^{+465}$ for the case of $f_{\max }=1000$ and $2048 \mathrm{~Hz}$, respectively, where $f_{\max }$ is the maximum frequency in the analysis. We also reanalyze the event with other waveform models: two post-Newtonian waveform models (TF2_PNTidal and TF2+_PNTidal), the TF2+_NRTidal model that is another NR calibrated waveform model, and its upgrade, the TF2+_NRTidalv2 model. While estimates of parameters other than $\tilde{\Lambda}$ are broadly consistent among various waveform models, our results indicate that estimates of $\tilde{\Lambda}$ depend on waveform models. However, the difference is smaller than the statistical error. For GW190425, we can only obtain little information on the binary tidal deformability. The systematic difference among the NR calibrated waveform models will become significant to measure $\tilde{\Lambda}$ as the number of detectors and events increase and sensitivities of detectors are improved.
\end{abstract}

DOI: 10.1103/PhysRevResearch.2.043039

\section{INTRODUCTION}

Binary-neutron-star (BNS) mergers are valuable laboratories for nuclear astrophysics. Matter effects influence the orbital evolution and gravitational radiation through the tidal interaction between the neutron stars (NSs) in the late inspiral phase. Additionally, the presence of material gives rise to electromagnetic emission primarily after the gravitational radiation. Because these signals depend on the properties of nuclear matter, their observations allow us to study nuclearmatter properties such as the equation of state (EOS) for NS matter.

GW170817 [1] and associated electromagnetic counterparts are used to derive various constraints on NS properties

\footnotetext{
*narikawa@icrr.u-tokyo.ac.jp

†uchikata@icrr.u-tokyo.ac.jp

${ }^{\ddagger}$ kkawa@icrr.u-tokyo.ac.jp

§kenta.kiuchi@aei.mpg.de

"kyutoku@tap.scphys.kyoto-u.ac.jp

IImshibata@yukawa.kyoto-u.ac.jp

**tagoshi@icrr.u-tokyo.ac.jp
}

Published by the American Physical Society under the terms of the Creative Commons Attribution 4.0 International license. Further distribution of this work must maintain attribution to the author(s) and the published article's title, journal citation, and DOI. and the underlying EOS. The existence of a blue component in the kilonova/macronova AT 2017gfo [2] might suggest that the merger remnant did not collapse promptly to a black hole. Thus, the maximum mass of the NS should not be as small as $\sim 2 M_{\odot}$ [3] and also the radii of high-mass NS may not be very small, e.g., the radius of the maximum-mass configuration is likely to be larger than $9.60 \mathrm{~km} \mathrm{[4]} \mathrm{(but} \mathrm{see} \mathrm{also} \mathrm{Ref.} \mathrm{[5]).} \mathrm{At}$ the same time, the short gamma-ray burst GRB 170817A [6] and the absence of magnetar-powered emission in AT 2017gfo suggest that the remnant NS collapsed early in the postmerger phase (but see also Refs. [7-10]). Accordingly, a maximum mass of $\gtrsim 2.3 M_{\odot}$ is also unlikely [3,11-14].

Tidal deformability extracted via cross-correlating gravitational-wave (GW) data of GW170817 with theoretical waveforms gives us more concrete information about the NS than electromagnetic counterparts. The LIGO-Virgo collaborations (LVC) initially put an upper limit on the binary tidal deformability $\tilde{\Lambda}$ of the binary as $\tilde{\Lambda} \lesssim 800$ with the prior on the dimensionless NS spin being chosen to be $|\chi| \leqslant 0.05$ [1]. This limit is later corrected to be $\tilde{\Lambda} \lesssim 900$ in Ref. [15], where the result of updated analysis is also reported as, e.g., $\tilde{\Lambda}=300_{-230}^{+420}$ for a particular set of assumptions. The constraint can be further improved by assuming the EOS to be common for both NS [16,17] (but see also Ref. [18]) as is also done in an independent analysis $[19,20]$. These constraints are used to investigate the NS EOS [21-23] as well as those for quark and hybrid stars [24-26]. While it has been claimed 
based on a limited number of numerical-relativity (NR) simulations that $\tilde{\Lambda} \gtrsim 400$ is necessary to account for the ejecta mass of $\approx 0.05 M_{\odot}$ required to explain AT 2017gfo [27] (see Ref. [28] for an updated analyses stating the bound on the tidal deformability in $323 \leqslant \tilde{\Lambda} \leqslant 776$ and Ref. [29], stating the similar bound to be in $302 \leqslant \tilde{\Lambda} \leqslant 860$ ), later systematic investigations reveal that this argument is premature [5].

Recently, the discovery of the second BNS merger event, GW190425, was reported [30]. This binary system is massive and it is intrinsically difficult to measure the tidal effect. LVC have reported that GW190425 constrains $\tilde{\Lambda}$ to be below 650 for the low-spin prior $(|\chi| \leqslant 0.05)$, after reweighting the posterior to derive approximately the result corresponding to a flat prior in $\tilde{\Lambda}$ [30]. While GW190425 does not carry novel information on the NS properties, multimessenger constraints on the NS EOS have been studied [31,32].

An accurate theoretical waveform template is crucial to extract accurately the tidal deformability of NSs from the observed GW data. For the early stage of the inspiral, the waveforms including the linear-order tidal effects derived by post-Newtonian (PN) calculation are useful $[33,34]$. However, the PN expansion becomes invalid as the orbit becomes relativistic and, thus, the error of the waveform becomes large in the late stage [35-38]. Such errors would cause the systematic bias in the parameter estimation, and it would be in particular problematic for estimating the tidal deformability because the tidal effects on the waveform become most significant just before the merger $[39,40]$. The effective-one-body (EOB) formalism can solve this problem by incorporating the higher-order PN correction by resummation techniques and calibrating them to NR waveforms [39,41-45]. Hence, employing waveform models with the higher-order PN correction calibrated to NR waveforms, such as the EOB formalism, is crucial for the data analysis.

Another approach to solve the problem is to adopt phenomenological models calibrated also to NR waveforms. Dietrich et al. have derived a gravitational waveform model, NRTidal, for BNSs based on high-precision NR simulations [46]. Improved reanalyses of GW170817 with more sophisticated waveform models calibrated to NR simulation of BNS merger have been performed employing such a model [15]. Indeed, it is pointed out that the value of the tidal deformability tends to be overestimated if the PN models are employed for the parameter estimation [1]. Recently, its upgrade, the NRTidalv2 model, which is calibrated to more precise NR waveforms, has been derived [47]. Kawaguchi et al. have also developed a model (hereafter the KyotoTidal model) for frequency-domain gravitational waveforms of inspiraling BNSs [48]. In particular, this model is derived independently from the NRTidal model employing different NR waveforms. Since the NRTidal model is to date the only NR calibrated waveform model that is used for parameter estimation of GWs from BNS mergers, the analysis comparing these three NR calibrated waveform models would help us to understand the systematic differences in resulting constraints on tidal deformability.

In this paper, we reanalyze the data around GW170817 and GW190425 against a NR calibrated waveform model, the TF2+_KyotoTidal model and present constraints on the binary tidal deformability. We also reanalyze the events with other waveform models: two PN (TF2_PNTidal and TF2+_PNTidal), TF2+_NRTidal, and TF2+_NRTidalv2 models. Here, TF2 is the abbreviation of TaylorF2, which is the PN waveform model for a point-particle part [49,50] and TF2+ [48] is a phenomenologically extended model of TF2.

The remainder of this paper is organized as follows. In Sec. II, we explain the methods for parameter estimation including waveform models used to reanalyze GW170817 and GW190425. In Sec. III, we present results of our analysis of GW170817 and a comparison of our analysis with the LVC analysis. In Sec. IV, we present results of GW190425 and discuss constraints on NS EOS by combining information obtained from GW170817 and GW190425. Section V is devoted to a summary. In the Appendix, we present an in-depth study of our results for GW170817 by separate analysis for the LIGO twin detectors to interpret the origin of the complex structure at the high $-\tilde{\Lambda}$ region for the posterior probability density function (PDF) of $\tilde{\Lambda}$ (see also Ref. [51]). Unless otherwise stated, we employ the units $c=G=1$, where $c$ and $G$ are the speed of light and the gravitational constant, respectively.

\section{PARAMETER ESTIMATION METHODS}

\section{A. Data and Bayesian inference}

We use Bayesian inference to reanalyze GW170817 and GW190425 with various waveform models that incorporate tidal effects in a different manner. Our analysis follows the one performed in our recent work [51], and uses the public data by LVC. ${ }^{1}$ We calculate the posterior PDF, $p(\vec{\theta} \mid \vec{s}(t), H)$, for the binary parameters $\vec{\theta}$ for the gravitational waveform model $H$ given the LIGO Hanford, LIGO Livingston, and Virgo data $s \overrightarrow{(t)}$ via

$$
p(\vec{\theta} \mid \vec{s}(t), H) \propto p(\vec{\theta} \mid H) p(\vec{s}(t) \mid \vec{\theta}, H) .
$$

$p(\vec{\theta} \mid H)$ is the prior for the binary parameters. The likelihood $p(\vec{s}(t) \mid \vec{\theta}, H)$ is evaluated by assuming stationarity and Gaussianity for the detector noise using the noise power spectrum density derived with Bayes Line. ${ }^{2}$ We compute PDFs by using stochastic sampling engine based on nested sampling $[52,53]$. Specifically, we use the parameter estimation software, LALINFERENCE [54,55], which is one of the software of LIGO Algorithm Library (LAL) software suite. We take the frequency range from $23 \mathrm{~Hz}$ for GW170817 and $19.4 \mathrm{~Hz}$ for GW190425 to $f_{\max }$. Here, the maximum frequency $f_{\max }$ is chosen from two values, $1000 \mathrm{~Hz}$ or $\min \left[f_{\mathrm{ISCO}}, f_{s} / 2\right]$, where $f_{\text {ISCO }}$ is twice the orbital frequency at the innermost stable circular orbit of a Schwarzschild black hole with total mass of the binary, and $f_{s}$ is the sampling rate of data. We set $f_{s}=4096 \mathrm{~Hz}$. The former choice is made because the TF2+_KyotoTidal model is calibrated in the frequency

\footnotetext{
${ }^{1}$ https://www.gw-openscience.org/catalog/GWTC-1-confident/ single/GW170817/ for Hanford and Virgo for GW170817, https://dcc.ligo.org/LIGO-T1700406/public for Livingston for GW170817, and https://dcc.ligo.org/LIGO-T1900685/public for GW190425.

${ }^{2}$ https://dcc.ligo.org/LIGO-P1900011/public
} 
TABLE I. Waveform models used to reanalyze GW170817 and GW190425. Our reference model, the TF2+_KyotoTidal model, incorporates TF2+ as the point-particle and spin parts, and NR calibrated tidal effects. The TF2 approximant employs the 3.5PN- and 3PN-order formulas for the phase and amplitude, respectively as the point-particle part, and treats aligned spins and incorporates 3.5PN-order formula in spin-orbit interactions, $2 \mathrm{PN}$-order formula in spin-spin, and self-spin interactions. TF2+ is the TF2 approximant supplemented with phenomenological higher-order PN terms calibrated to SEOBNRv2 for the point-particle part. The TF2+_NRTidal model is another model whose tidal effects are calibrated to NR. The TF2+_NRTidalv2 model is the upgrade of the TF2+_NRTidal model. The TF2_PNTidal and TF2+_PNTidal models employ the PN tidal-part phase formula.

\begin{tabular}{lcccc}
\hline \hline \multirow{2}{*}{ Model name } & \multicolumn{2}{c}{ Point-particle part } & & Tidal part \\
\cline { 2 - 3 } & Amplitude & Phase & Amplitude & Phase \\
\hline TF2_PNTidal & $3 \mathrm{PN}$ & $3.5 \mathrm{PN}$ & $5+1 \mathrm{PN}$ & $5+2.5 \mathrm{PN}$ \\
TF2+_PNTidal & $6 \mathrm{PN}$ & $6 \mathrm{PN}$ & $5+1 \mathrm{PN}$ & $5+2.5 \mathrm{PN}$ \\
TF2+_KyotoTidal & $6 \mathrm{PN}$ & $6 \mathrm{PN}$ & Polynomial & Nonlinear \\
TF2+_NRTidal & $6 \mathrm{PN}$ & $6 \mathrm{PN}$ & Padé approximation \\
TF2+_NRTidalv2 & $6 \mathrm{PN}$ & $6 \mathrm{PN}$ & Padé approximation & Padé approximation \\
\hline \hline
\end{tabular}

range of $10-1000 \mathrm{~Hz}$. The latter choice corresponds to the assumption that the inspiral stage is terminated at the smaller of $f_{\text {ISCO }}$ and $f_{s} / 2$. In this work, we represent the latter choice by $f_{\max }=2048 \mathrm{~Hz}$ for simplicity.

\section{B. Waveform models for inspiraling BNSs}

We use various analytic frequency-domain waveform models for the inspiral phase, all of which are constructed based on the PN formulas. The features of each waveform model are summarized in Table I. The Fourier transform of the gravitational waveform can be written as

$$
\tilde{h}(f)=A(f) e^{i \Psi(f)},
$$

where the amplitude $A(f)$ and the phase $\Psi(f)$ can be decomposed into the point-particle evolution, the spin effects, and the tidal effects as

$$
A(f)=A_{\text {point-particle }}(f)+A_{\text {spin }}(f)+A_{\text {tidal }}(f)
$$

and

$$
\Psi(f)=\Psi_{\text {point-particle }}(f)+\Psi_{\text {spin }}(f)+\Psi_{\text {tidal }}(f) .
$$

We use TF2 [49,50] and phenomenologically extended model of TF2, called TF2+ (see Ref. [48] and below) as BBH baseline, which consists of point-particle and spin parts. Here, the 3.5PN-order formula for the phase and 3PN-order formula for the amplitude are employed as the point-paticle part of TF2 [56]. For TF2+, both the phase and amplitude of the point-particle part are extended to the $6 \mathrm{PN}$ order by fitting the SEOBNRv2 model [57,58].

All waveform models used in our parameter estimation analyses assume that the spins of component stars are aligned with the orbital angular momentum, and incorporate 3.5PN-order formula in couplings between the orbital angular momentum and the component spins [59], 2PN-order formula in point-mass spin-spin, and self-spin interactions [60,61].

During the BNS inspiral, at the leading order, the induced quadrupole moment tensor $Q_{i j}$ is proportional to the external tidal field tensor $\mathcal{E}_{i j}$ as $Q_{i j}=-\lambda \mathcal{E}_{i j}$. The information about the NS EOS can be quantified by the tidal deformability parameter $\lambda[33,62]$. The leading-order tidal contribution to the GW phase evolution (relative 5PN order) is governed by the symmetric contribution of NS tidal deformation, characterized by the binary tidal deformability [33]

$$
\tilde{\Lambda}=\frac{16}{13} \frac{\left(m_{1}+12 m_{2}\right) m_{1}^{4} \Lambda_{1}+\left(m_{2}+12 m_{1}\right) m_{2}^{4} \Lambda_{2}}{\left(m_{1}+m_{2}\right)^{5}},
$$

which is a mass-weighted linear combination of the tidal deformability of the both components, where $m_{1,2}$ is the component mass and $\Lambda_{1,2}$ is the dimensionless tidal deformability parameter of each star $\Lambda_{1,2}=\lambda_{1,2} / m_{1,2}^{5}$. The antisymmetric contribution $\delta \tilde{\Lambda}$ terms introduced in Refs. [35,38] are always subdominant on the tidal effects to the GW phase and the symmetric contribution $\tilde{\Lambda}$ terms dominate [35,38]. In this paper, we ignore the $\delta \tilde{\Lambda}$ contribution.

The TF2_PNTidal and TF2+_PNTidal models denote the waveform models employing $\mathrm{TF} 2$ and $\mathrm{TF} 2+$ as the $\mathrm{BBH}$ baseline, respectively. Both the TF2_PNTidal and the TF2+_PNTidal models employ the $2.5 \mathrm{PN}$-order (relative $5+$ 2.5PN-order) tidal-part phase formula [39]

$$
\begin{aligned}
\Psi_{\text {tidal }}^{\text {PNTidal }}= & \frac{3}{128 \eta}\left[-\frac{39}{2} \tilde{\Lambda} x^{5 / 2}\left(1+\frac{3115}{1248} x-\pi x^{3 / 2}\right.\right. \\
& \left.\left.+\frac{28024205}{3302208} x^{2}-\frac{4283}{1092} \pi x^{5 / 2}\right)\right],
\end{aligned}
$$

where $x=\left[\pi M_{\mathrm{tot}}(1+z) f\right]^{2 / 3}$ is the dimensionless PN parameter, $M_{\mathrm{tot}}=m_{1}+m_{2}$ is the total mass, $\eta=m_{1} m_{2} /\left(m_{1}+\right.$ $\left.m_{2}\right)^{2}$ is the symmetric mass ratio, and $z$ is the source redshift. The tidal-part amplitude for both TF2_PNTidal and TF2+_PNTidal models employs the $5+1 \mathrm{PN}$-order amplitude formula given by [39]

$$
A_{\text {tidal }}^{\text {PNTidal }}=\sqrt{\frac{5 \pi \eta}{24}} \frac{M_{\text {tot }}^{2}(1+z)^{2}}{d_{L}} \tilde{\Lambda} x^{-7 / 4}\left(-\frac{27}{16} x^{5}-\frac{449}{64} x^{6}\right),
$$

where $d_{L}$ is the luminosity distance to the source.

The TF2+_KyotoTidal model is a NR calibrated waveform model for the inspiral phase of BNS mergers [48,63]. The TF2+_KyotoTidal model employs TF2+ as the BBH baseline and extends the 2.5PN-order (relative $5+2.5 \mathrm{PN}$-order) tidal-part phase formula [39] by multiplying $\tilde{\Lambda}$ by a nonlinear correction to model the tidal part of the $\mathrm{GW}$ phase. The 
functional forms of the tidal-part phase are

$$
\begin{aligned}
& \Psi_{\text {tidal }}^{\text {KyotoTidal }} \\
& =\frac{3}{128 \eta}\left[-\frac{39}{2} \tilde{\Lambda}\left(1+a \tilde{\Lambda}^{2 / 3} x^{p}\right)\right] x^{5 / 2} \\
& \times\left(1+\frac{3115}{1248} x-\pi x^{3 / 2}+\frac{28024205}{3302208} x^{2}-\frac{4283}{1092} \pi x^{5 / 2}\right),
\end{aligned}
$$

where $a=12.55$ and $p=4.240$. The tidal-part amplitude is extended by adding the higher-order PN tidal effects to Eq. (7) as

$$
\begin{aligned}
A_{\text {tidal }}^{\text {KyotoTidal }}= & \sqrt{\frac{5 \pi \eta}{24}} \frac{M_{\text {tot }}^{2}(1+z)^{2}}{d_{L}} \tilde{\Lambda} x^{-7 / 4} \\
& \times\left(-\frac{27}{16} x^{5}-\frac{449}{64} x^{6}-b x^{r}\right),
\end{aligned}
$$

where $b=4251$ and $r=7.890$. In the KyotoTidal model, the hybrid waveforms constructed from high-precision NR waveforms and the SEOBNRv2T waveforms [45,57,58,64,65] are used for model calibration in the frequency range of 10-1000 Hz. The phase difference between the TF2+_KyotoTidal model and the hybrid waveforms is smaller than $0.1 \mathrm{rad}$ up to $1000 \mathrm{~Hz}$ for $300 \lesssim \tilde{\Lambda} \lesssim 1900$ and for the mass ratio $q=m_{2} / m_{1} \leqslant 1$ between 0.73 and 1 [48]. In Ref. [48], it is shown that the mismatch between the TF2+_KyotoTidal model and the hybrid waveforms is always smaller than $1.1 \times 10^{-5}$ in the frequency range of 10-1000 Hz.

The NRTidal model is another approach to describe tidal effects calibrated to NR waveforms [46]. The TF2+_NRTidal model employs $\mathrm{TF} 2+$ as the $\mathrm{BBH}$ baseline. For the tidal effects, this model extends the linear-order effects by effectively adding the higher-order PN terms of the tidal contribution to the GW phase. As shown in Ref. [46], the expression of the tidal phase is given by the form of a rational function:

$$
\Psi_{\text {tidal }}^{\mathrm{NRTidal}}=\frac{3}{128 \eta}\left[-\frac{39}{2} \tilde{\Lambda} x^{5 / 2} \frac{1+\tilde{n}_{1} x+\tilde{n}_{3 / 2} x^{3 / 2}+\tilde{n}_{2} x^{2}+\tilde{n}_{5 / 2} x^{5 / 2}}{1+\tilde{d}_{1} x+\tilde{d}_{3 / 2} x^{3 / 2}}\right],
$$

where $\tilde{d}_{1}=\tilde{n}_{1}-3115 / 1248$, the other parameters are $\left(\tilde{n}_{1}, \tilde{n}_{3 / 2}, \tilde{n}_{2}, \tilde{n}_{5 / 2}\right)=(-17.428,31.867,-26.414,62.362)$ and $\tilde{d}_{3 / 2}=$ 36.089. We do not consider the tidal-part amplitude for this model following the original form [46].

The TF2+_NRTidalv2 model is an upgrade of the TF2+_NRTidal model [47]. Specifically, they derive a new expression for the tidal phase which is calibrated to more accurate NR waveforms as

$$
\Psi_{\text {tidal }}^{\mathrm{NRTidalv2}}=\frac{3}{128 \eta}\left[-\frac{39}{2} \tilde{\Lambda} x^{5 / 2} \frac{1+\tilde{n}_{1}^{\prime} x+\tilde{n}_{3 / 2}^{\prime} x^{3 / 2}+\tilde{n}_{2}^{\prime} x^{2}+\tilde{n}_{5 / 2}^{\prime} x^{5 / 2}+\tilde{n}_{3}^{\prime} x^{3}}{1+\tilde{d}_{1}^{\prime} x+\tilde{d}_{3 / 2}^{\prime} x^{3 / 2}+\tilde{d}_{2}^{\prime} x^{2}}\right],
$$

with $\tilde{n}_{1}^{\prime}=\tilde{c}_{1}^{\prime}+\tilde{d}_{1}^{\prime}, \tilde{n}_{3 / 2}^{\prime}=\left(\tilde{c}_{1}^{\prime} \tilde{c}_{3 / 2}^{\prime}-\tilde{c}_{5 / 2}^{\prime}-\tilde{c}_{3 / 2}^{\prime} \tilde{d}_{1}^{\prime}+\tilde{n}_{5 / 2}^{\prime}\right) / \tilde{c}_{1}^{\prime}$, $\tilde{n}_{2}^{\prime}=\tilde{c}_{2}^{\prime}+\tilde{c}_{1}^{\prime} \tilde{d}_{1}^{\prime}+\tilde{d}_{2}^{\prime}, \quad \tilde{d}_{3 / 2}^{\prime}=-\left(\tilde{c}_{5 / 2}^{\prime}+\tilde{c}_{3 / 2}^{\prime} \tilde{d}_{1}^{\prime}-\tilde{n}_{5 / 2}^{\prime}\right) / \tilde{c}_{1}^{\prime}$, where the known coefficients are $\tilde{c}_{1}^{\prime}=3115 / 1248, \tilde{c}_{3 / 2}^{\prime}=$ $-\pi, \quad \tilde{c}_{2}^{\prime}=28024205 / 3302208, \quad \tilde{c}_{5 / 2}^{\prime}=-4283 \pi / 1092$, and the fitting coefficients are $\tilde{n}_{5 / 2}^{\prime}=90.550822$, $\tilde{n}_{3}^{\prime}=-60.253578, \quad \tilde{d}_{1}^{\prime}=-15.111208, \quad \tilde{d}_{2}^{\prime}=8.0641096$. They also introduce the tidal amplitude

$$
\begin{aligned}
A_{\text {tidal }}^{\mathrm{NRTidalv2}}= & \sqrt{\frac{5 \pi \eta}{24} \frac{M_{\mathrm{tot}}^{2}(1+z)^{2}}{d_{L}} \tilde{\Lambda} x^{-7 / 4}} \\
& \times\left(-\frac{27}{16} x^{5}\right) \frac{1+\frac{449}{108} x+\frac{22672}{9} x^{2.89}}{1+d x^{4}},
\end{aligned}
$$

where $d=13477.8$. Although the new phase model NRTidalv2, introduced in Ref. [47], includes higher-order spin-squared and spin-cubed terms with their associated spininduced moments, we do not add them in this work.

In Fig. 1, we show differences in the phase evolution of tidal part among the KyotoTidal, NRTidal, NRTidalv2, and PNTidal models. A difference in the treatment of the tidal effects makes different $\tilde{\Lambda}$ dependence. The tidal phase normalized by the leading (relative $5 \mathrm{PN}$-order) tidal phase formula for the KyotoTidal model depends on the binary tidal deformability $\tilde{\Lambda}$ due to the nonlinear correction. Since the NRTidal, NRTidalv2, and PNTidal models employ the linearorder effects of the tidal deformability, they are independent of
$\tilde{\Lambda}$ when normalized by the leading tidal effect. Figure 1 shows good agreement between the TF2+_KyotoTidal model and the TF2+_NRTidalv 2 model for $\tilde{\Lambda} \simeq 1000$ below $1000 \mathrm{~Hz}$ as suggested in Ref. [47]. The NRTidal model gives the largest phase shift, the second is the NRTidalv2 model, the third is the KyotoTidal model, and the PNTidal model gives the smallest, for $\tilde{\Lambda} \leqslant 1000$, up to $\sim 1000 \mathrm{~Hz}$. The TF2+_KyotoTidal model is calibrated only up to $1000 \mathrm{~Hz}$ and overestimates tidal effects at frequencies above $1000 \mathrm{~Hz}$. The KyotoTidal model gives the largest phase shift at frequency above $1200 \mathrm{~Hz}$ for $\tilde{\Lambda}=1000$, and larger phase shift than the one for the NRTidalv 2 model at frequency above about 1000 and $1400 \mathrm{~Hz}$ for $\tilde{\Lambda}=1000$ and 400 , respectively.

\section{Source parameters}

The source parameters and their prior probability distributions are chosen to follow those adopted in our recent work [51], and we mention specific choices made in this work.

For GW170817, we fix the sky location to the position of AT $2017 \mathrm{gfo}$, which is an electromagnetic counterpart of GW170817 [66], and estimates of the remaining source parameters. Specifically, we estimate the luminosity distance to the source $d_{L}$, the binary inclination $\theta_{\mathrm{JN}}$, which is the angle between the total angular momentum and the line of sight, the polarization angle $\psi$, the coalescence time $t_{c}$, the phase at the coalescence time $\phi_{c}$, component masses $m_{1,2}$, where 


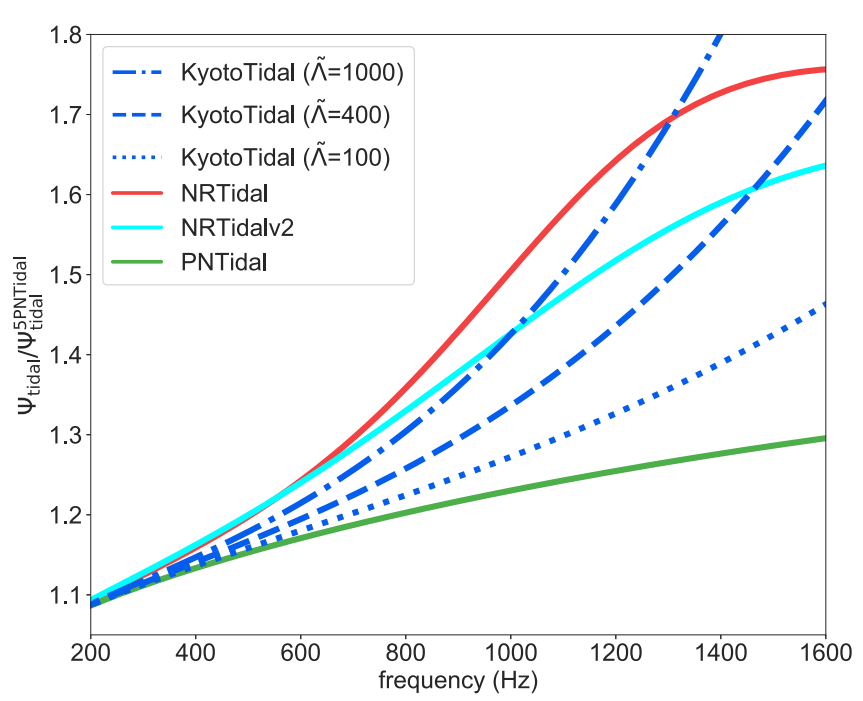

FIG. 1. Tidal phase in the frequency domain normalized by the leading, Newtonian (relative 5PN-order) tidal phase formula. Here, we use $\left(m_{1}, m_{2}\right)=\left(1.35 M_{\odot}, 1.35 M_{\odot}\right)$. We show $\tilde{\Lambda}=1000$ (dotted-dashed, blue), 400 (dashed, blue), and 100 (dotted, blue) for the KyotoTidal model. The NRTidal model (solid, red), the NRTidalv2 model (solid, cyan), and the $5+2.5 \mathrm{PN}$-order tidal-part phase formula, PNTidal (solid, green), are also presented, which are independent of $\tilde{\Lambda}$ when normalized by the leading tidal phase.

we assume $m_{1} \geqslant m_{2}$, the orbit-aligned dimensionless spin components of the stars $\chi_{1,2}$ where $\chi_{1,2}=c S_{1,2} /\left(G m_{1,2}^{2}\right)$ is the orbit-aligned dimensionless spin component of the stars with $S_{1,2}$ are the magnitudes of the spin angular momenta of the components, and the binary tidal deformability $\tilde{\Lambda}$.

For GW170817, we assume a uniform distribution as the detector-frame component mass prior $m_{1,2} \sim$ $U[0.83,7.7] M_{\odot}$ with an additional constraint on the detectorframe chirp mass $\mathcal{M}^{\text {det }}:=\mathcal{M}(1+z) \sim U[1.184,2.168] M_{\odot}$, where the chirp mass is the best estimated mass parameter defined by $\mathcal{M}=\left(m_{1} m_{2}\right)^{3 / 5}\left(m_{1}+m_{2}\right)^{-1 / 5}$. The prior range for $\mathcal{M}^{\text {det }}$ is the same as that used for LVC analysis [15]. The impact of wider prior range for $\mathcal{M}^{\text {det }}$ on parameter estimation is negligible. We assume a uniform prior on the spin magnitudes and we enforce $\chi_{1,2} \sim U[-0.05,0.05]$. This prior range of spin is consistent with the observed population of known BNSs that will merge within the Hubble time $[67,68]$, and is referred to as the low-spin prior for the LVC analysis [15]. We assume a uniform prior on the binary tidal deformability, with $\tilde{\Lambda} \sim U[0,3000]$.

For GW190425, we also estimate the sky location of the source with an isotropic prior. We assume the detector-frame component mass prior $m_{1,2} \sim U[1.0,5.0] M_{\odot}$ and the spin and the binary tidal deformability priors are the same as the ones for GW170817.

\section{RESULTS OF GW170817}

\section{A. Source properties other than the tidal deformability}

In this section, we show validity of our analysis as a sanity check by comparison with the LVC results. Figure 2 shows the marginalized posterior PDFs of parameters other than the tidal deformability for various waveform models for $f_{\max }=1000 \mathrm{~Hz}$. Table II presents the $90 \%$ credible intervals of the luminosity distance $d_{L}$, the binary inclination $\theta_{\mathrm{JN}}$, mass parameters (the component masses $m_{1,2}$, the detectorframe chirp mass $\mathcal{M}^{\text {det }}$, the source-frame chirp mass $\mathcal{M}$, the total mass $M_{\text {tot }}$, and the mass ratio $q$ ), and the effective spin parameter $\chi_{\text {eff }}=\left(m_{1} \chi_{1}+m_{2} \chi_{2}\right) / M_{\text {tot }}$, which is the most measurable combination of spin components, estimated using various waveform models [69,70]. The source-frame chirp mass is derived by assuming a value of the Hubble constant $H_{0}=69 \mathrm{~km} \mathrm{~s}^{-1} \mathrm{Mpc}^{-1}$ (a default value in LAL adopted from Planck 2013 results [71]).

For comparison of our analysis with the results of the previous LVC analysis [15,72], we also analyze GW170817 by using the restricted TF2 approximant as the waveform model with $5+1 \mathrm{PN}$-order tidal-part phase formula. This model has the $\mathrm{BBH}$ baseline whose amplitude is constructed only from the Newtonian-order point-particle evolution [49,50,59-61] and is implemented in LALINFERENCE. We checked that estimates of parameters other than the tidal deformability we obtained by using the restricted TF2 model are broadly consistent with the LVC results presented in Refs. [15,72].

The estimates of parameters other than the tidal deformability presented in Fig. 2 and Table II show the absence of significant systematic difference associated with a difference among waveform models for both $\mathrm{BBH}$ baseline and tidal parts. The posterior PDFs of these parameters for $f_{\max }=2048 \mathrm{~Hz}$ agree approximately with the ones for $f_{\max }=$ $1000 \mathrm{~Hz}$ as illustrated for the TF2_PNTidal model in Fig. 2. This is due to the fact that the parameters other than the tidal deformability are mainly measured from information at low-frequency region [39] and terms up to 3.5PN order of the point-particle part for the phase are the same among different waveforms. On the other hand, the tidal deformability is measured primarily from information at high-frequency region as discussed in the next section. ${ }^{3}$

\section{B. Posterior of binary tidal deformability}

Before presenting our results obtained with various waveform models, we first compare our results obtained by using the restricted TF2 model that incorporates the $5+1 \mathrm{PN}$-order tidal-part phase with those from the LVC analysis [15] as a sanity check. The restricted TF2 model version used by LVC analysis includes no amplitude corrections and has a uniform prior on the component tidal deformability, with $\Lambda_{1,2} \sim$ $U[0,5000]$. While our result of $90 \%$ credible symmetric and highest posterior density (HPD) intervals on $\tilde{\Lambda}$ are $347_{-243}^{+564}$ and $347_{-295}^{+453}$, respectively, for restricted TF2 with $5+1 \mathrm{PN}$ order tidal-part phase, low-spin prior $\left(\left|\chi_{1,2}\right| \leqslant 0.05\right)$, and $f_{\max }=2048 \mathrm{~Hz}$, the LVC report $\tilde{\Lambda}=340_{-240}^{+580}$ and $340_{-290}^{+490}$, respectively, in [15]. Here, uniform priors in $\Lambda_{1}$ and $\Lambda_{2}$ are adopted in both analyses, and the posterior of $\tilde{\Lambda}$ is divided by

\footnotetext{
${ }^{3}$ We note that the spin-induced quadrupole moments can affect largely estimates of the component spins and mass ratio for large NSs' spins, and combination of the effects of the spin-induced quadrupole moments and the tidal deformability is important to investigate NS EOS as shown in Ref. [73].
} 

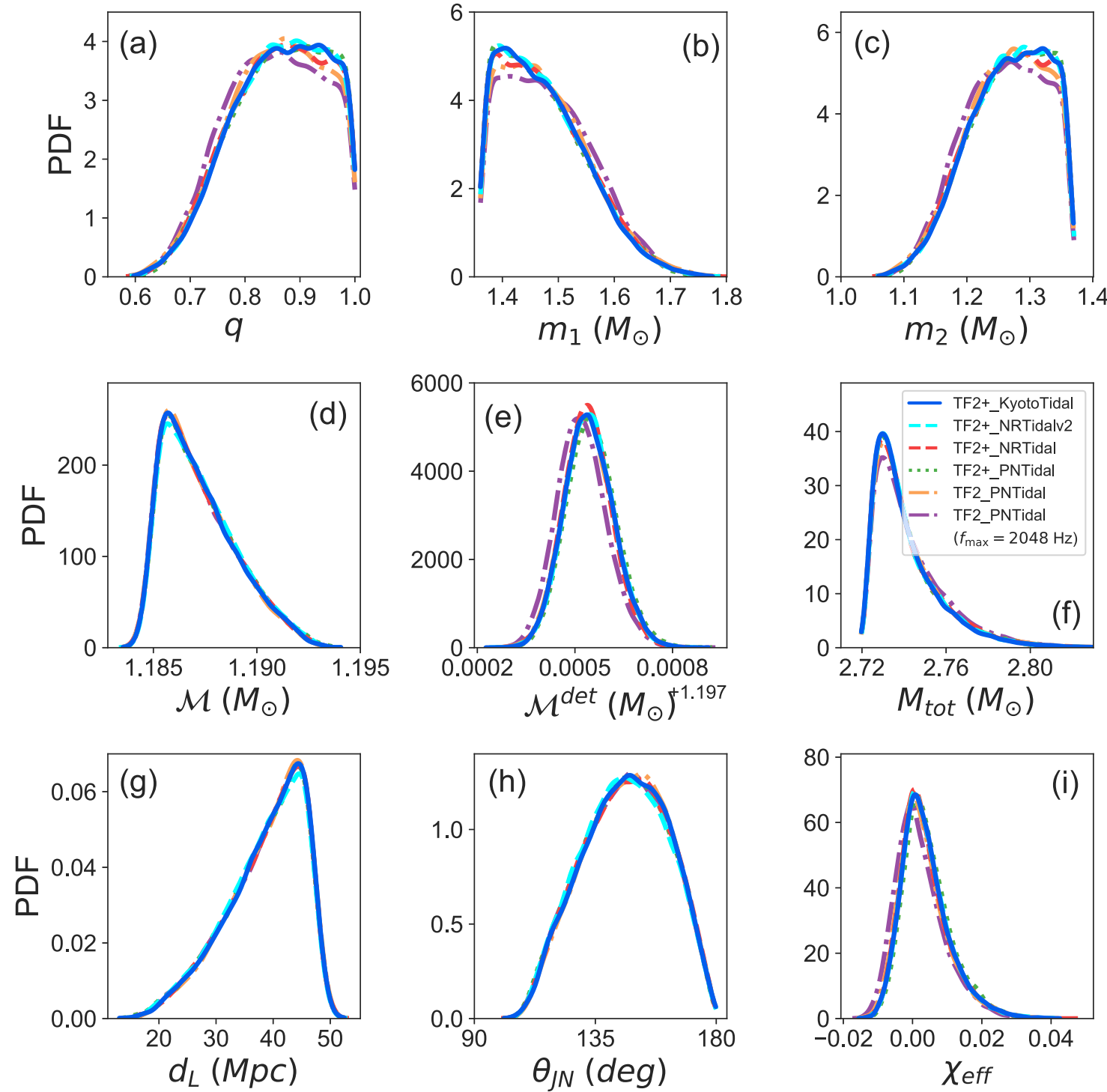

FIG. 2. Marginalized posterior PDFs of various parameters for GW170817 derived by various waveform models. The blue, cyan, red, green, and orange curves correspond to the TF2+_KyotoTidal, TF2+_NRTidalv2, TF2+_NRTidal, TF2+_PNTidal, and TF2_PNTidal models, respectively. The top-left, top-middle, top-right, middle-left, center, middle-right, bottom-left, bottom-middle, and bottom-right panels show (a) the mass ratio $q$, (b) the primary mass $m_{1}$, (c) the secondary mass $m_{2}$, (d) the source-frame chirp mass $\mathcal{M}$, (e) the detector-frame chirp mass $\mathcal{M}^{\text {det }}$, (f) the total mass $M_{\text {tot }}$, (g) the luminosity distance to the source $d_{L}$, (h) the inclination angle $\theta_{\mathrm{JN}}$, and (i) the effective spin parameter $\chi_{\text {eff }}$, respectively. Here, we show the distribution for $f_{\max }=1000 \mathrm{~Hz}$, except for the TF2_PNTidal model, for which the intervals for both $f_{\max }=1000$ and $2048 \mathrm{~Hz}$ are given.

its prior determined by those of other parameters following [15] to derive approximate results for the case of a uniform prior on $\tilde{\Lambda}$. The closeness of the inferred credible ranges indicates that our analysis successfully reproduces the results derived by the LVC. If we assume a uniform prior on $\tilde{\Lambda}$ from the beginning, $90 \%$ credible symmetric and HPD intervals on $\tilde{\Lambda}$ are $316_{-224}^{+504}$ and $316_{-291}^{+367}$, respectively, for restricted TF2 with $5+1$ PN-order tidal-part phase.

Figure 3 shows the marginalized posterior PDFs for the binary tidal deformability $\tilde{\Lambda}$ for various waveform models with both (a) $f_{\max }=1000$ and (b) $2048 \mathrm{~Hz}$. The corresponding $90 \%$ credible intervals are presented in Table III. We caution that the TF2+_KyotoTidal model is calibrated only up to $1000 \mathrm{~Hz}$ and can overestimate tidal effects at frequencies above $1000 \mathrm{~Hz}$. Thus, the results for $f_{\max }=2048 \mathrm{~Hz}$ should be regarded as only a reference.

For $f_{\max }=1000 \mathrm{~Hz}$ [see Fig. 3(a)], the peak values of $\tilde{\Lambda}$ are located between 400 and 500 and the $90 \%$ credible intervals do not extend to $\gtrsim 900$ for NR calibrated waveform models: the TF2+_KyotoTidal, TF2+_NRTidalv2, and TF2+_NRTidal models. Our results show that the posterior of binary tidal deformability for GW170817 depends on waveform models. The TF2+_KyotoTidal, TF2+_NRTidal, TF2+_NRTidalv2, and TF2+_PNTidal models are constructed from the same $\mathrm{BBH}$ baseline, $\mathrm{TF} 2+$, but with different tidal descriptions. Therefore, a difference of estimates among these waveform models reflects directly their different tidal description. The TF2+_NRTidal model gives the smallest median 
TABLE II. $90 \%$ credible interval of the luminosity distance $d_{L}$, the binary inclination $\theta_{\mathrm{JN}}$, mass parameters, and the effective spin parameter $\chi_{\text {eff }}$ for GW170817 estimated using various waveform models. We show $10 \%-100 \%$ regions of the mass ratio with the upper limit $q=1$ imposed by definition, and those of $m_{1}$ and $m_{2}$ are given accordingly. We give symmetric $90 \%$ credible intervals, i.e., $5 \%-95 \%$, for the other parameters with the median as a representative value.

\begin{tabular}{lccccc}
\hline \hline & TF2_PNTidal & TF2+_PNTidal & TF2+_KyotoTidal & TF2+_NRTidal & TF2+_NRTidalv2 \\
\hline Luminosity distance $d_{L}(\mathrm{Mpc})$ & $40.0_{-14.4}^{+7.3}$ & $39.8_{-14.7}^{+7.5}$ & $39.9_{-14.6}^{+7.3}$ & $39.9_{-14.5}^{+7.4}$ & $39.6_{-14.6}^{+7.7}$ \\
Binary inclination $\theta_{\mathrm{JN}}($ degree $)$ & $147_{-32}^{+24}$ & $146_{-27}^{+24}$ & $147_{-28}^{+24}$ & $147_{-27}^{+24}$ & $146_{-27}^{+25}$ \\
Detector-frame chirp mass $\mathcal{M}^{\text {det }}\left(M_{\odot}\right)$ & $1.1975_{-0.0001}^{+0.0001}$ & $1.1975_{-0.0001}^{+0.0001}$ & $1.1975_{-0.0001}^{+0.0001}$ & $1.1975_{-0.0001}^{+0.0001}$ & $1.1975_{-0.0001}^{+0.0001}$ \\
Source-frame chirp mass $\mathcal{M}\left(M_{\odot}\right)$ & $1.187_{-0.002}^{+0.004}$ & $1.187_{-0.002}^{+0.004}$ & $1.187_{-0.002}^{+0.004}$ & $1.187_{-0.002}^{+0.004}$ & $1.187_{-0.002}^{+0.004}$ \\
Primary mass $m_{1}\left(M_{\odot}\right)$ & $(1.36,1.59)$ & $(1.36,1.58)$ & $(1.36,1.58)$ & $(1.36,1.59)$ & $(1.36,1.58)$ \\
Secondary mass $m_{2}\left(M_{\odot}\right)$ & $(1.18,1.37)$ & $(1.18,1.37)$ & $(1.18,1.37)$ & $(1.18,1.37)$ & $(1.18,1.37)$ \\
Total mass $M_{\text {tot }}:=m_{1}+m_{2}\left(M_{\odot}\right)$ & $2.74_{-0.01}^{+0.04}$ & $2.74_{-0.01}^{+0.04}$ & $2.74_{-0.01}^{+0.04}$ & $2.74_{-0.01}^{+0.04}$ & $2.74_{-0.01}^{+0.04}$ \\
Mass ratio $q:=m_{2} / m_{1}$ & $(0.74,1.00)$ & $(0.74,1.00)$ & $(0.75,1.00)$ & $(0.75,1.00)$ & $(0.75,1)$ \\
Effective spin $\chi_{\text {eff }}$ & $0.002_{-0.009}^{+0.015}$ & $0.003_{-0.009}^{+0.015}$ & $0.003_{-0.008}^{+0.014}$ & $0.002_{-0.008}^{+0.015}$ & $0.003_{-0.008}^{+0.014}$ \\
\hline \hline
\end{tabular}

value on $\tilde{\Lambda}$ of 403 , the second is the TF2+_NRTidalv2 model of 445 , the third is the TF2+_KyotoTidal model of 481 , and the TF2+_PNTidal model gives the largest one of 569. This order is derived from the order of the phase shift of different waveform models for a given value of $\tilde{\Lambda}=400$, up to about $1400 \mathrm{~Hz}$ as shown in Fig. 1. The tendency to give smaller estimated values for NR calibrated waveform models than for PN waveform models are consistent with previous results derived in Ref. [74] (see also Ref. [75] for the detailed study of systematic biases associated with spin effects). The TF2+_PNTidal and TF2_PNTidal models are constructed from the same tidal part and the different point-particle part. A difference in the posterior PDFs of estimated $\tilde{\Lambda}$ between these models is very small for $f_{\max }=1000 \mathrm{~Hz}$. This result shows that the higher-order point-particle terms do not significantly affect the estimate of the binary tidal deformability of GW170817 for $f_{\max }=1000 \mathrm{~Hz}$. (See Ref. [76] for systematic

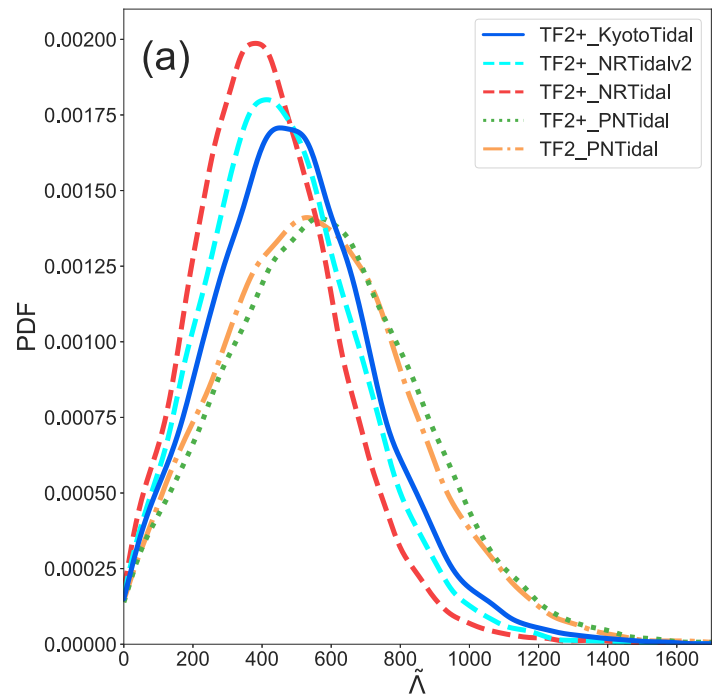

study on the binary tidal deformability by injection of signals with incomplete baselines).

For $f_{\max }=2048 \mathrm{~Hz}$ [see Fig. 3(b)], the peak values of $\tilde{\Lambda}$ are located between 250 and 400 and the $90 \%$ credible intervals do not extend to $\gtrsim 850$ for NR calibrated waveform models. The widths of symmetric $90 \%$ credible intervals for $f_{\max }=2048 \mathrm{~Hz}$ are narrower than those for $f_{\max }=1000 \mathrm{~Hz}$, by about $7 \%$ for the TF2+_KyotoTidal model, $4 \%$ for the TF2+_NRTidal model, 5\% for the TF2+_NRTidalv2 model, $13 \%$ for the TF2+_PNTidal model, and about 5\% for the TF2_PNTidal model, as shown in Table III. This decrease in the width of the interval is consistent with the fact that higher-frequency data are more informative to measure $\tilde{\Lambda}$ [39]. The peak values of the posterior PDFs of $\tilde{\Lambda}$ tend to decrease as $f_{\max }$ increases for all waveform models as shown in Fig. 3. The order of peak values of $\tilde{\Lambda}$ for the different waveform models that incorporate the same BBH baseline,

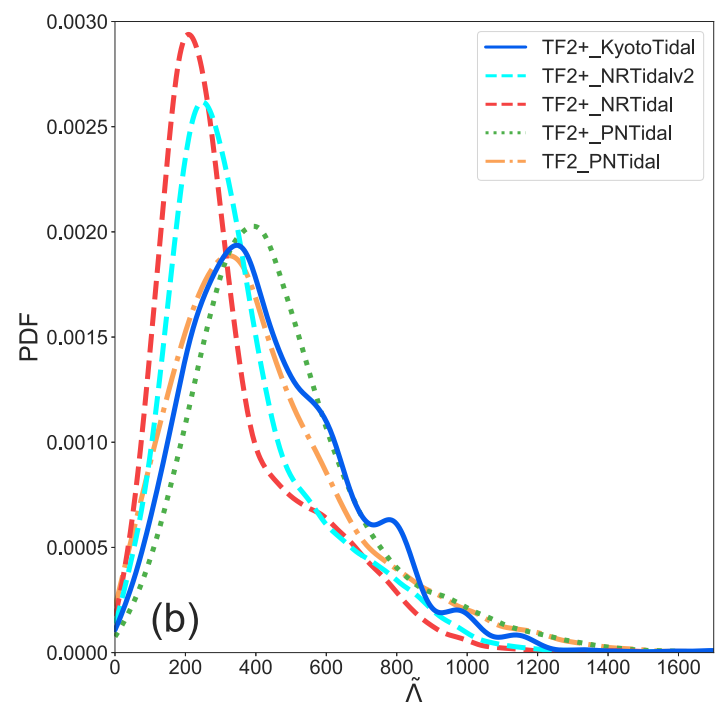

FIG. 3. Marginalized posterior PDFs of binary tidal deformability $\tilde{\Lambda}$ for GW170817, estimated by various waveform models, for (a) $f_{\text {max }}=$ 1000 and (b) $2048 \mathrm{~Hz}$. The blue, cyan, red, green, and orange curves correspond to the TF2+_KyotoTidal, TF2+_NRTidalv2, TF2+_NRTidal, TF2+_PNTidal, and TF2_PNTidal models, respectively. The corresponding 90\% credible intervals are presented in Table III. 
TABLE III. 90\% credible interval of the binary tidal deformability $\tilde{\Lambda}$ for GW170817 for various waveform models. We report both the symmetric $90 \%$ credible interval (symmetric) and the $90 \%$ highest-posterior-density (HPD) intervals, for both $f_{\max }=1000$ (left side) and $2048 \mathrm{~Hz}$ (right side), where the median is shown as a representative value.

\begin{tabular}{|c|c|c|c|c|}
\hline \multirow[b]{2}{*}{ Model } & \multicolumn{2}{|c|}{$f_{\max }=1000 \mathrm{~Hz}$} & \multicolumn{2}{|c|}{$f_{\max }=2048 \mathrm{~Hz}$} \\
\hline & Symmetric & HPD & Symmetric & HPD \\
\hline TF2_PNTidal & $548_{-415}^{+500}$ & $548_{-463}^{+433}$ & $376_{-284}^{+584}$ & $376_{-353}^{+442}$ \\
\hline TF2+_PNTidal & $569_{-431}^{+496}$ & $569_{-470}^{+441}$ & $428_{-280}^{+540}$ & $428_{-353}^{+414}$ \\
\hline TF2+_KyotoTidal & $481_{-359}^{+436}$ & $481_{-402}^{+379}$ & $402_{-279}^{+465}$ & $402_{-316}^{+419}$ \\
\hline TF2+_NRTidal & $403_{-299}^{+378}$ & $403_{-337}^{+328}$ & $267_{-180}^{+491}$ & $267_{-228}^{+409}$ \\
\hline TF2+_NRTidalv2 & $445_{-330}^{+412}$ & $445_{-370}^{+357}$ & $312_{-208}^{+498}$ & $312_{-263}^{+407}$ \\
\hline
\end{tabular}

$\mathrm{TF} 2+$, is not affected by varying $f_{\max }$ as shown in Fig. 3. This is explained by the same reason as that for $f_{\max }=1000 \mathrm{~Hz}$. We note that $1400 \mathrm{~Hz}$ approximately corresponds to $f_{\mathrm{ISCO}}$ for estimated mass range. The TF2_PNTidal model gives a slightly smaller peak value than the TF2+_KyotoTidal model. This cannot be explained only by the feature of the tidal part as shown in Fig. 1. This might be due to the effects of the higher-order point-particle terms or the fact that the data at frequencies above $1000 \mathrm{~Hz}$ are dominated by the detector's noise. The difference in the posterior PDFs of estimated $\tilde{\Lambda}$ between the TF2+_PNTidal and TF2_PNTidal models for $f_{\max }=2048 \mathrm{~Hz}$ is larger than that for $f_{\max }=1000 \mathrm{~Hz}$ (see Fig. 3 and Table III). This is due to the effects of higher-order point-particle terms as discussed in Refs. [36,76].

\section{RESULTS OF GW190425 AND NS EOS}

We reanalyze data of the second BNS merger event, GW190425, using three waveform models: TF2+_KyotoTidal, TF2+_NRTidalv2, and TF2+_PNTidal models. We present marginalized posterior PDFs for source parameters of GW190425 in Fig. 4 and corresponding 90\% credible interval in Table IV. The estimates of parameters other than $\tilde{\Lambda}$ presented in Fig. 4 and Table IV are broadly consistent with the LVC results presented in Ref. [30] and show the absence of significant systematic difference among different waveform models. The posterior PDFs of these parameters for $f_{\max }=2048 \mathrm{~Hz}$ agree approximately with the ones for $f_{\max }=1000 \mathrm{~Hz}$ as illustrated for the TF2+_PNTidal model in Fig. 4.
Figure 5 shows marginalized posterior PDFs of $\tilde{\Lambda}$ for GW190425 with three waveform models. While this figure indicates that there is a small difference between $\mathrm{PN}$ and NR calibrated models, only a tiny difference is found between two NR calibrated models. The posterior PDF of $\tilde{\Lambda}$ has a large value around $\tilde{\Lambda}=0$ and this fact implies that no significant tidal effect is detected as found in Ref. [30]. HPD upper limit on the binary tidal deformability is $\tilde{\Lambda} \leqslant 610$ for the TF2+_KyotoTidal model for $f_{\max }=1000 \mathrm{~Hz}$. The posterior PDF of $\tilde{\Lambda}$ for the TF2+_KyotoTidal model with $f_{\max }=2048 \mathrm{~Hz}$ is bimodal. Investigation of the secondary peak's origin remains as a future work, but it may result from the nonlinear tidal terms $\propto x^{p}$ of this model, which increase rapidly at $\gtrsim 1000 \mathrm{~Hz}$ for which the calibration by the hybrid waveforms is not performed.

In order to discuss constraints on NS EOS by combining information obtained from GW170817 and GW190425, we plot prediction of various NS EOS on posterior of the binary tidal deformability and binary's chirp radius, which is a conveniently scaled dimensionful radiuslike parameter [38]. Figure 6 shows 50\% and 90\% credible regions in (a) the $\tilde{\Lambda}-\mathcal{M}$ plane and (b) the $\mathcal{M}-\mathcal{R}$ plane, for GW170817 and GW190425, where $\mathcal{R}=2 \mathcal{M} \tilde{\Lambda}^{1 / 5}$ is the binary's chirp radius. Five colored curves are posteriors predicted by various NS EOS models: MS1 [77], H4 [78], MPA1 [79], APR4 [80], and WFF1 [81]. For these plots, we use the masses uniformly distributed in the mass ratio range $0.7 \leqslant$ $q \leqslant 1$, which include the $90 \%$ credible regions of mass posteriors for GW170817 and GW190425. Our results using TF2+_KyotoTidal model show that the allowed chirp ra-

TABLE IV. Source properties for GW190425 using TF2+_PNTidal, TF2+_KyotoTidal, and TF2+_NRTidalv2 models for $f_{\max }=1000 \mathrm{~Hz}$ for the low-spin prior $\left(\left|\chi_{1,2}\right| \leqslant 0.05\right)$. For $\tilde{\Lambda}$, we show HPD upper limits.

\begin{tabular}{lccc}
\hline \hline & TF2+_PNTidal & TF2+_KyotoTidal & TF2+_NRTidalv2 \\
\hline Luminosity distance $d_{L}(\mathrm{Mpc})$ & $159_{-74}^{+67}$ & $159_{-73}^{+67}$ & $158_{-73}^{+67}$ \\
Detector-frame chirp mass $\mathcal{M}{ }^{\text {det }}\left(M_{\odot}\right)$ & $1.4867_{-0.0003}^{+0.0003}$ & $1.4867_{-0.0003}^{+0.003}$ & $1.4867_{-0.0003}^{+0.0003}$ \\
Source-frame chirp mass $\mathcal{M}\left(M_{\odot}\right)$ & $1.44_{-0.02}^{+0.02}$ & $1.44_{-0.02}^{+0.02}$ & $1.44_{-0.02}^{+0.02}$ \\
Primary mass $m_{1}\left(M_{\odot}\right)$ & $(1.62,1.90)$ & $(1.61,1.90)$ & $(1.61,1.90)$ \\
Secondary mass $m_{2} M_{\odot}$ & $(1.44,1.69)$ & $(1.44,1.69)$ & $(1.44,1.69)$ \\
Total mass $M_{\text {tot }}:=m_{1}+m_{2}\left(M_{\odot}\right)$ & $3.3_{-0.1}^{+0.1}$ & $3.3_{-0.1}^{+0.1}$ & $3.3_{-0.1}^{+0.1}$ \\
Mass ratio $q:=m_{2} / m_{1}$ & $(0.8,1.0)$ & $(0.8,1.0)$ & $(0.8,1.0)$ \\
Effective spin $\chi_{\text {eff }}$ & $0.010_{-0.012}^{+0.015}$ & $0.009_{-0.012}^{+0.015}$ & $0.009_{-0.012}^{+0.015}$ \\
Binary tidal deformability $\tilde{\Lambda}$ & $\leqslant 700$ & $\leqslant 610$ & $\leqslant 546$ \\
\hline \hline
\end{tabular}



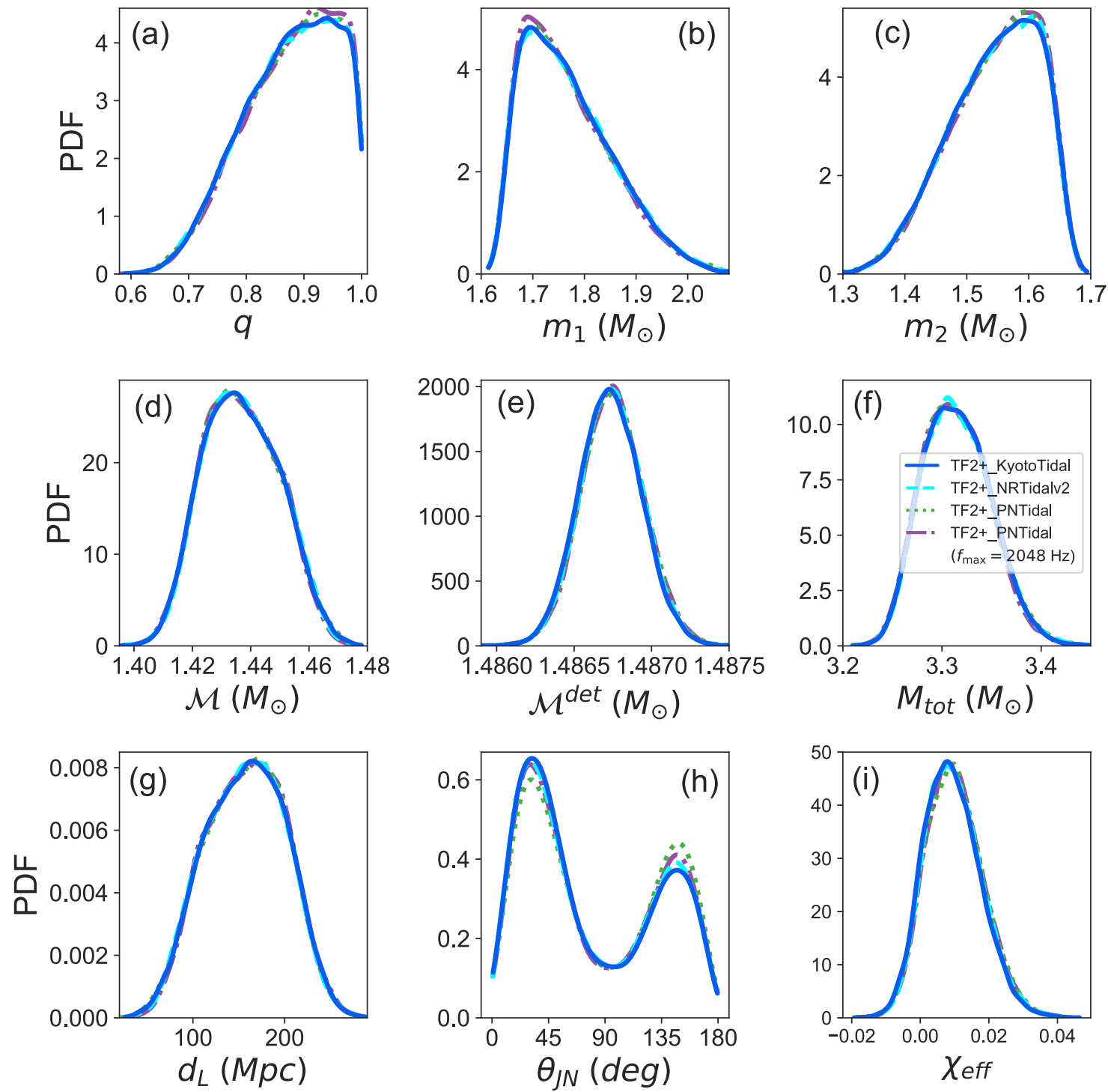

FIG. 4. Marginalized posterior PDFs of source parameters for GW190425 using TF2+_PNTidal, TF2+_KyotoTidal, and TF2+_NRTidalv2 models for the low-spin prior $\left(\left|\chi_{1,2}\right| \leqslant 0.05\right)$. Here, we show the distribution for $f_{\max }=1000 \mathrm{~Hz}$, except for the TF2 + PNTidal model, for which the intervals for both $f_{\max }=1000$ and $2048 \mathrm{~Hz}$ are given.

dius range is $10 \mathrm{~km} \lesssim \mathcal{R} \lesssim 14 \mathrm{~km}$ for the chirp mass $\mathcal{M} \simeq$ $1.2 M_{\odot}$. In particular, the MS1 and H4 models lie outside the $90 \%$ credible region for GW170817, while they are not disfavored from GW190425, which is consistent with the LVC results presented in Refs. [1,15-17].

\section{SUMMARY}

We reanalyze GW170817 and GW190425 with a NR calibrated waveform model, the TF2+_KyotoTidal model, which has been developed independently from the one used in previous studies by LVC. The TF2+_KyotoTidal model is calibrated in the frequency range of $10-1000 \mathrm{~Hz}$ to hybrid waveforms composed of high-precision NR waveforms and the SEOBNRv2T waveforms, and reproduces the phase of the hybrid waveforms within $0.1 \mathrm{rad}$ error up to $1000 \mathrm{~Hz}$. In the TF2+_KyotoTidal model, the nonlinear effects of the tidal deformability are incorporated. We also reanalyze the events with other waveform models: two PN waveform models (TF2 PNTidal and TF2+ PNTidal), the TF2+ NRTidal model that is another NR calibrated waveform model, and its upgrade, the TF2+_NRTidalv2 model.

We compare parameter estimation results with those by different tidal waveform models. For GW170817, we do not find any significant systematic differences for extraction of source parameters other than the binary tidal deformability using different waveform models. By contrast, we find the significant systematics in determining $\tilde{\Lambda}$. Specifically, we reconfirm that the PN model tends to overestimate $\tilde{\Lambda}$ compared to the NR calibrated waveform models as shown in Ref. [74] and, in addition, the estimates of $\tilde{\Lambda}$ depend on NR calibrated waveform models for $f_{\max }=1000 \mathrm{~Hz}$ although the difference is smaller than the statistical uncertainties.

Our results for GW170817 indeed indicate that $\tilde{\Lambda}$ is constrained more tightly for $f_{\max }=2048 \mathrm{~Hz}$ than for $f_{\max }=$ $1000 \mathrm{~Hz}$. For the TF2+_KyotoTidal model, the $90 \%$ symmet- 

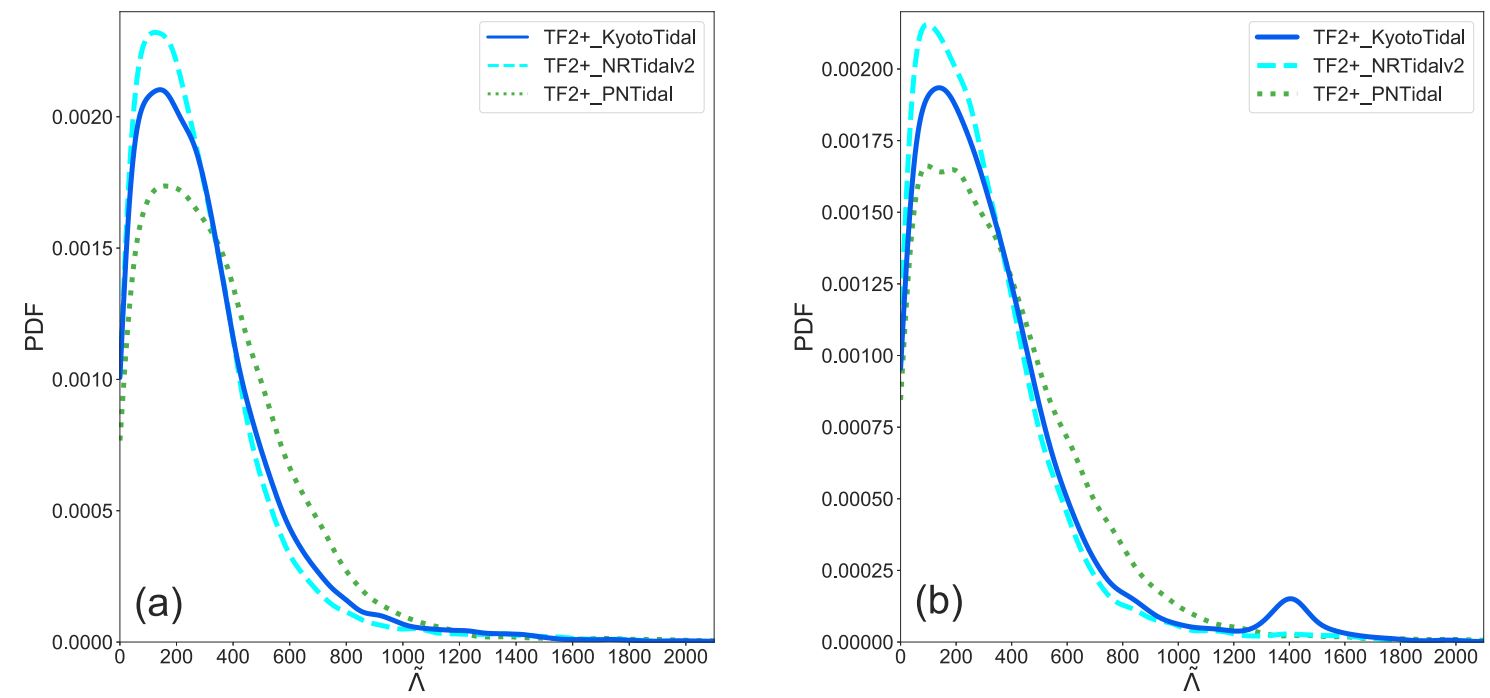

FIG. 5. Marginalized posterior PDFs of $\tilde{\Lambda}$ for GW190425 using TF2+_PNTidal, TF2+_KyotoTidal, and TF2+_NRTidalv2 models for the low-spin prior $\left(\left|\chi_{1,2}\right| \leqslant 0.05\right)$ for (a) $f_{\max }=1000$ and (b) $2048 \mathrm{~Hz}$.

ric interval of $\tilde{\Lambda}$ for $f_{\max }=2048 \mathrm{~Hz}$ is about $7 \%$ narrower than that for $f_{\max }=1000 \mathrm{~Hz}$. Although the credible interval of $\tilde{\Lambda}$ becomes narrower as the $f_{\max }$ increases, the TF2+_KyotoTidal model is calibrated only up to $1000 \mathrm{~Hz}$. Since higher-frequency data are more informative for $\tilde{\Lambda}$ [39], it is important to improve current waveform models at high frequencies above $1000 \mathrm{~Hz}$ to accurately determine $\tilde{\Lambda}$ from the GW data, toward third-generation detector era.

For the second BNS merger event, GW190425, we use three waveform models: TF2+_KyotoTidal, TF2+_NRTidalv2, and TF2+_PNTidal models. Similarly to GW170817, we do not find any significant systematic differences for extraction of source parameters other than the binary tidal deformability among different waveform models.

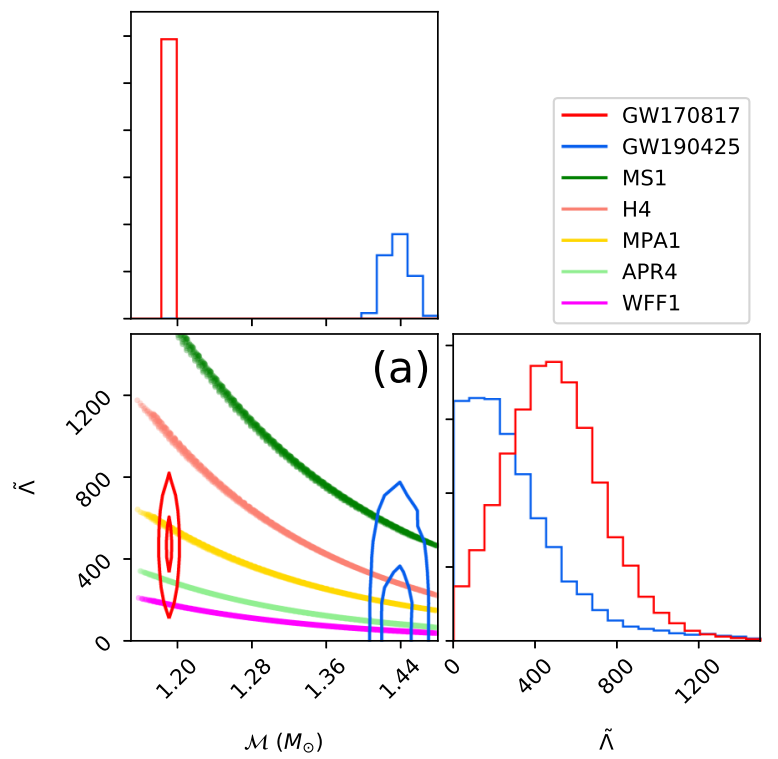

This binary system is massive and it is intrinsically difficult to measure the tidal effect. While our results show that the $90 \%$ credible interval of $\tilde{\Lambda}$ for the PN waveform model is slightly wider than for NR models, we do not find essential difference in the constraints for $\tilde{\Lambda}$ obtained by the different waveform models.

We discuss constraints on NS EOS models by combining information obtained from GW170817 and GW190425. Our results using TF2+_KyotoTidal model show that the chirp radius $\mathcal{R}$ is constrained between about 10 and $14 \mathrm{~km}$ for the chirp mass $\mathcal{M} \simeq 1.2 M_{\odot}$. By using an independent waveform model (TF2+_KyotoTidal model) and independent analysis, we obtain the results consistent with the LVC's one: a low SNR event from a massive BNS like GW190425 cannot

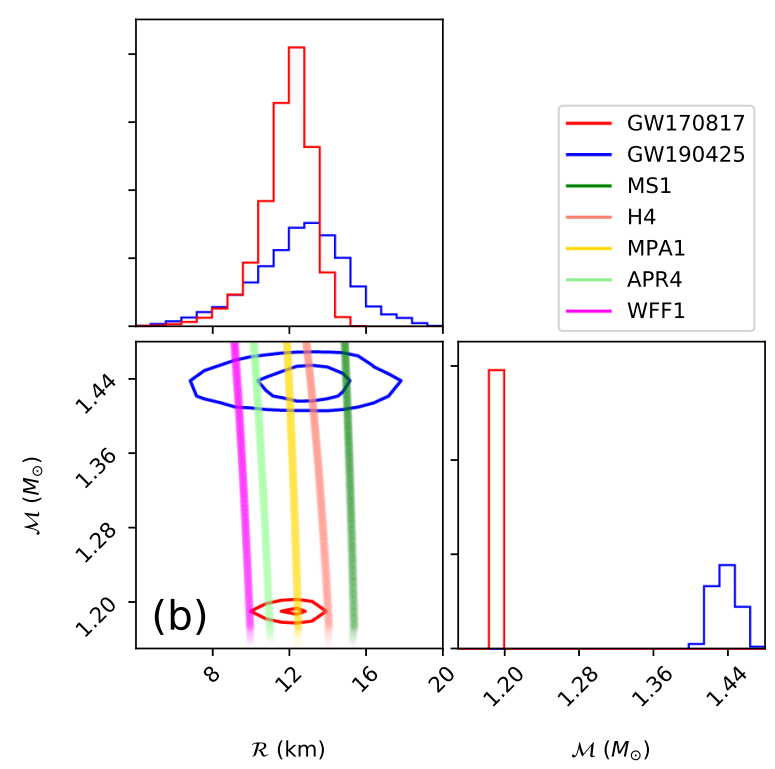

FIG. 6. $50 \%$ and $90 \%$ credible regions in (a) the $\tilde{\Lambda}-\mathcal{M}$ plane and (b) the $\mathcal{M}-\mathcal{R}$ plane obtained from GW170817 (red) and GW190425 (blue) events using TF2+_KyotoTidal model for $f_{\max }=1000 \mathrm{~Hz}$ for the low-spin prior $\left(\left|\chi_{1,2}\right| \leqslant 0.05\right)$. Five colored curves are calculated in the mass ratio range $0.7 \leqslant q \leqslant 1$ with various EOS models: MS1, H4, MPA1, APR4, and WFF1. 

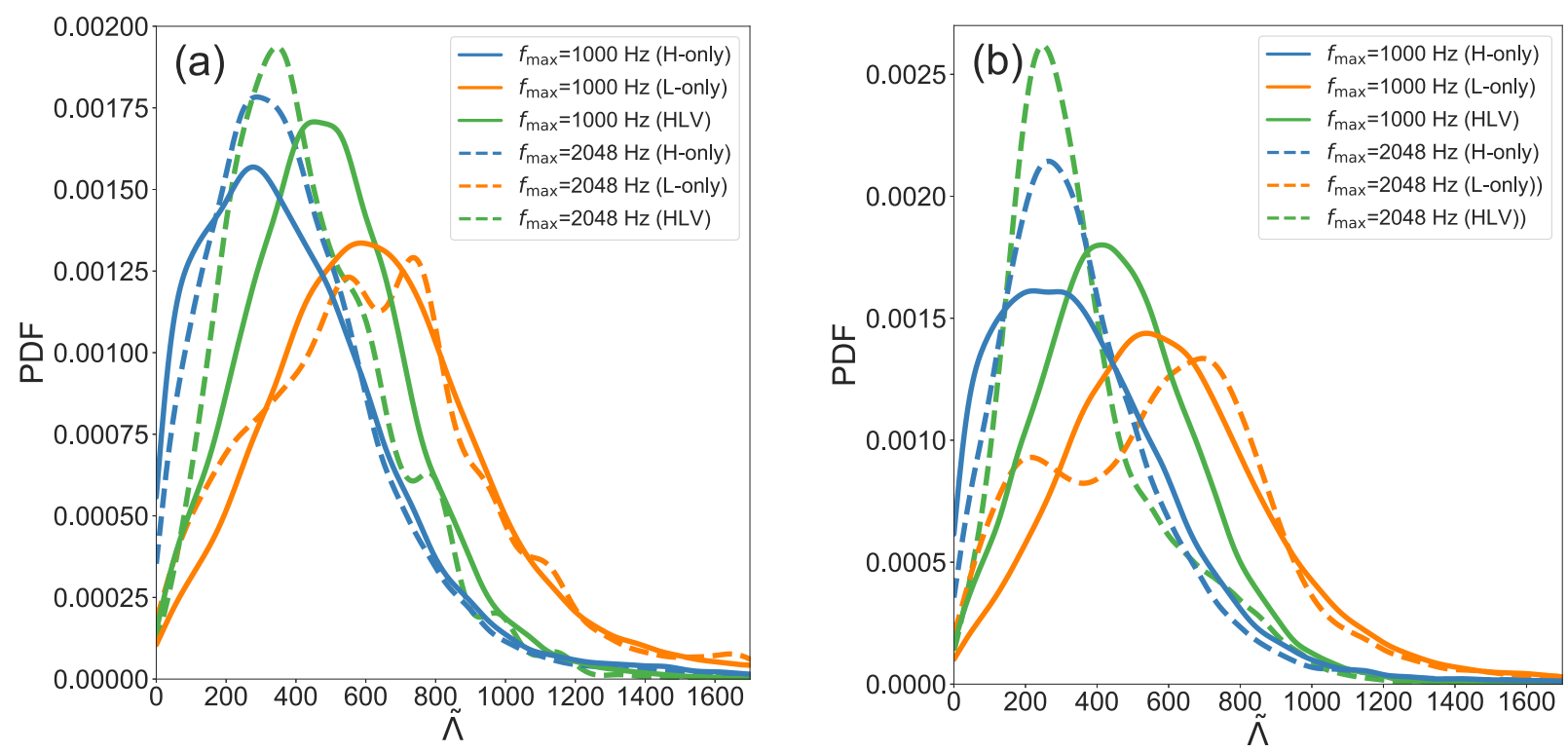

FIG. 7. Marginalized posterior PDFs of binary tidal deformability $\tilde{\Lambda}$ for GW170817 derived by data of different detector combinations with both $f_{\max }=1000$ (solid) and 2048 (dashed) Hz for (a) the TF2+_KyotoTidal and (b) the TF2+_NRTidalv2 models. The distribution derived by the Hanford-only data (blue), that by the Livingston-only data (orange), and that by combined data of advanced LIGO twin detectors and advanced Virgo (green, denoted by HLV) are presented. For $f_{\max }=2048 \mathrm{~Hz}$, a multimodal (bump) structure at high $\tilde{\Lambda}$ for the TF2+_KyotoTidal (TF2+_NRTidalv2) model appear due to Livingston data.

contribute very much to constraining the NS EOS as shown in Ref. [30]. As the number of BNS merger events increases and sensitivities of detectors are improved, the systematic differences will become significant.

\section{ACKNOWLEDGMENTS}

We thank J. Veitch for very helpful explanation of LALINFERENCE and C. van den Broeck for useful discus- sions. This work is supported by Japanese Society for the Promotion of Science (JSPS) KAKENHI Grants No. JP15K05081, No. JP16H02183, No. JP16H06341, No. JP16H06342, No. JP17H01131, No. JP17H06133, No. JP17H06358, No. JP17H06361, No. JP17H06364, No. JP18H01213, No. JP18H04595, No. JP18H05236, and No. JP19K14720, and by a post-K project hp180179. This work is also supported by JSPS Core-to-Core Program A. Advanced Research Networks and by the joint research program of the Institute for
Cosmic Ray Research, University of Tokyo, Computing Infrastructure Project of KISTI-GSDC in Korea, and Computing Infrastructure ORION in Osaka City University. T. Narikawa is supported in part by a Grant-in-Aid for JSPS Research Fellows, and he also thanks hospitality of van den Broeck's group during his stay at Nikhef. K. Kawaguchi was supported in part by JSPS overseas research fellowships. We are also grateful to the LIGO-Virgo collaborations for the public release of GW data of GW170817. This research has made use of data, software, and web tools obtained from the Gravitational Wave Open Science Center [82], a service of LIGO Laboratory, the LIGO Scientific Collaboration, and the Virgo Collaboration. LIGO is funded by the U. S. National Science Foundation. Virgo is funded by the French Centre National de la Recherche Scientifique (CNRS), the Italian Istituto Nazionale di Fisica Nucleare (INFN), and the Dutch Nikhef, with contributions by Polish and Hungarian institutes.

TABLE V. 90\% credible interval of binary tidal deformability, for GW170817 $\tilde{\Lambda}$, with the TF2+_KyotoTidal (left side) and the TF2+_NRTidalv2 (right side) models, for different detector data and the maximum frequency $f_{\max }$. The upper group shows the symmetric intervals and the lower shows the HPD intervals, where the median is shown as a representative value for both groups.

\begin{tabular}{|c|c|c|c|c|}
\hline \multirow[b]{2}{*}{$f_{\max }$} & \multicolumn{2}{|c|}{ TF2+_KyotoTidal } & \multicolumn{2}{|c|}{ TF2+_NRTidalv2 } \\
\hline & Hanford only & Livingston only & Hanford only & Livingston only \\
\hline & \multicolumn{4}{|c|}{ Symmetric } \\
\hline $1000 \mathrm{~Hz}$ & $357_{-311}^{+568}$ & $618_{-447}^{+637}$ & $333_{-291}^{+514}$ & $582_{-413}^{+586}$ \\
\hline \multirow[t]{2}{*}{$2048 \mathrm{~Hz}$} & $362_{-295}^{+514}$ & $607_{-482}^{+658}$ & $320_{-253}^{+481}$ & $589_{-487}^{+549}$ \\
\hline & \multicolumn{4}{|c|}{ HPD } \\
\hline $1000 \mathrm{~Hz}$ & $357_{-357}^{+414}$ & $618_{-523}^{+502}$ & $333_{-333}^{+378}$ & $582_{-484}^{+477}$ \\
\hline $2048 \mathrm{~Hz}$ & $362_{-352}^{+378}$ & $607_{-557}^{+511}$ & $320_{-305}^{+355}$ & $589_{-555}^{+399}$ \\
\hline
\end{tabular}




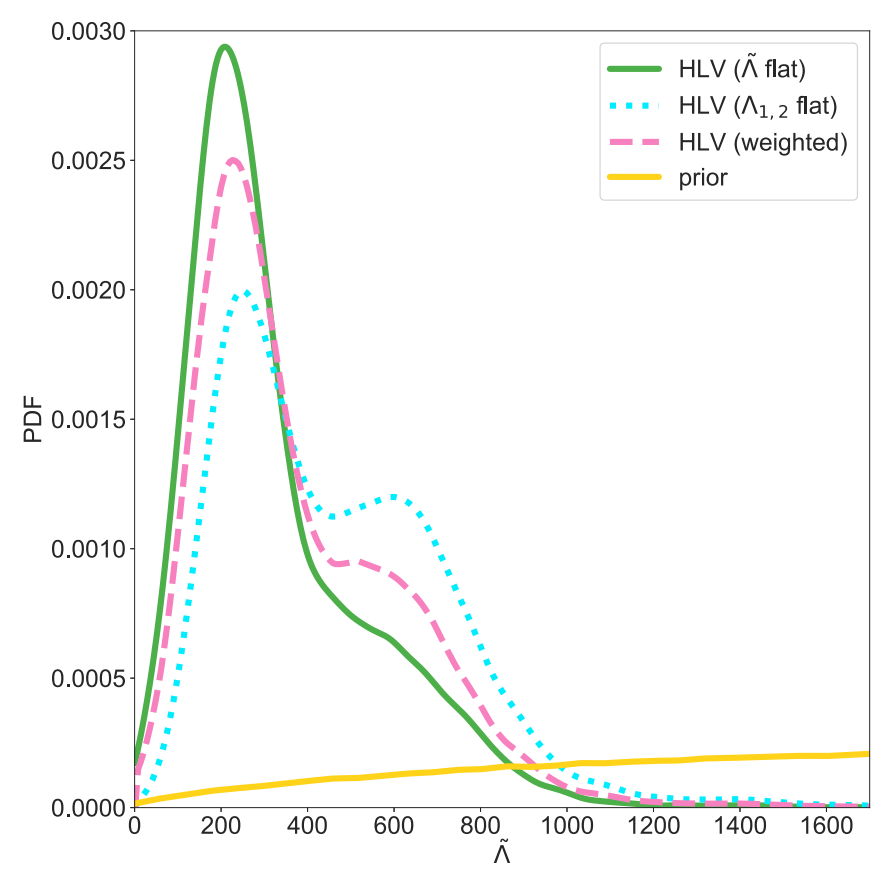

FIG. 8. Dependence of the marginalized posterior PDFs of $\tilde{\Lambda}$ on different priors in $\tilde{\Lambda}$ for GW170817 for the TF2+_NRTidal model with $f_{\max }=2048 \mathrm{~Hz}$. In addition to PDF of $\tilde{\Lambda}$ for uniform priors in $\Lambda_{1}$ and $\Lambda_{2}$ (dotted, cyan), we show the PDF for "weighted" prior (dashed, magenta), which is weighted by dividing the original prior (also shown by solid yellow curve) and the PDF for a uniform prior in $\tilde{\Lambda}$ (solid, green).

\section{APPENDIX: SEPARATE ANALYSIS FOR THE LIGO TWIN DETECTORS FOR GW170817}

There is a multimodal structure at the high- $\tilde{\Lambda}$ region in the posterior PDF of $\tilde{\Lambda}$ for GW170817 for the TF2+_KyotoTidal model and a bump structure for the TF2+_NRTidal and TF2+_NRTidalv2 models for $f_{\max }=2048 \mathrm{~Hz}$ as shown in Fig. 3(b). In this Appendix, we present an in-depth study to interpret these features by separate analysis for the LIGO twin detectors for GW170817. Figure 7 shows marginalized posterior of $\tilde{\Lambda}$ derived by separate analysis for the Hanford and Livingston detectors with both $f_{\max }=1000$ and $2048 \mathrm{~Hz}$ for (a) the TF2+_KyotoTidal model and (b) the TF2+_NRTidalv2 model. Table V shows corresponding $90 \%$ credible intervals of $\tilde{\Lambda}$.

In the case of the TF2+_KyotoTidal model, Fig. 7(a) suggests that the origin of the bump at the high- $\tilde{\Lambda}$ region for $f_{\max }=2048 \mathrm{~Hz}$ for the HLV combined data is as follows. On the one hand, for the Livingston data, the unimodal distribution for $f_{\max }=1000 \mathrm{~Hz}$, whose peak is at about 600 , is separated into a bimodal distribution for $f_{\max }=2048 \mathrm{~Hz}$ that is constructed from twin peaks, a low- $\tilde{\Lambda}$ bump, and a few high- $\tilde{\Lambda}$ bumps. On the other hand, for the Hanford data, the unimodal distribution for $f_{\max }=1000 \mathrm{~Hz}$, whose peak is at low $-\tilde{\Lambda}$ region, shrinks for $f_{\max }=2048 \mathrm{~Hz}$. As a result, for $f_{\max }=2048 \mathrm{~Hz}$, the remaining high- $\tilde{\Lambda}$ peak for the Livingston data produces the bump for the HLV combined data. Moreover, a few high- $\tilde{\Lambda}$ bumps in the case of HLV combined data for $f_{\max }=2048 \mathrm{~Hz}$ are inherited from the bumps of the Livingston-only data, which are associated with the highfrequency data. The location of the low- $\tilde{\Lambda}$ bump derived by the Livingston-only data is close to the peak of $\tilde{\Lambda}$ of about 250 derived by the Hanford-only data.

In the case of the TF2+_NRTidalv2 model, as shown in Fig. 7(b), a bump at the high- $\tilde{\Lambda}$ region in the case of HLV combined data for $f_{\max }=2048 \mathrm{~Hz}$ are inherited from the peak of the Livingston-only data, $\tilde{\Lambda} \sim 750$.

While a bimodal distribution appears in the posterior PDF of $\tilde{\Lambda}$ with the SEOBNRv4_ROM_NRTidal model in the case of LVC analysis as shown in Fig. 11 in [15], a small high- $\tilde{\Lambda}$ bump at $\tilde{\Lambda} \sim 600$ appears in that with the TF2+_NRTidal model presented for $f_{\max }=2048 \mathrm{~Hz}$ in Fig. 3(b). Here, the SEOBNRv4_ROM_NRTidal model is constructed from the SEOBNRv4 model $[83,84]$ as the BBH baseline and the NRTidal model as the tidal part. Supplementary analysis with the TF2+_NRTidal model as shown in Fig. 8 demonstrates that the different priors in $\tilde{\Lambda}$ (one uniform and one nonuniform) make such different distribution between our analysis and the LVC analysis. The LVC used "weighted" prior. In this prior, they assume uniform priors in $\Lambda_{1}$ and $\Lambda_{2}$, and weight the posterior of $\tilde{\Lambda}$ by dividing by its prior determined by those of other parameters [15]. "Weighted" prior approximately corresponds to imposing a uniform prior on $\tilde{\Lambda}$. Figure 8 shows the dependence of the results on different priors in $\tilde{\Lambda}$, " $\Lambda_{1,2}$ flat," "weighted," and " $\tilde{\Lambda}$ flat" for the TF2+_NRTidal model with $f_{\max }=2048 \mathrm{~Hz}$. This figure demonstrates that the distribution for " $\Lambda_{1,2}$ flat" and "weighted" prior tends to be a bimodal rather than a high- $\tilde{\Lambda}$ bump.

In Ref. [51], it is found that there is a discrepancy in the estimates of binary tidal deformability of GW170817 between the Hanford and Livingston detectors of Advanced LIGO by using the restricted TaylorF2 waveform model. Figure 7 shows that the discrepancy is enhanced with sophisticated waveform models (the TF2+_KyotoTidal and TF2+_NRTidalv2 models). While the two distributions in the cases of the Hanford-only and Livingston-only data seem to be consistent with each other and also consistent with what we would expect from noise realization (see, e.g., Ref. [38]), the results that the width of the $90 \%$ credible interval for the Livingston-only data does not shrink as $f_{\max }$ increases indicate that the Livingston's high-frequency data are not very useful to determine the tidal deformability for GW170817.
[1] B. P. Abbott, R. Abbott, T. D. Abbott, F. Acernese, K. Ackley, C. Adams, T. Adams, P. Addesso, R. X. Adhikari, V. B. Adya et al. (LIGO Scientific and Virgo Collaborations), GW170817: Observation of Gravitational Waves from a Binary Neutron Star Inspiral, Phys. Rev. Lett. 119, 161101 (2017).
[2] B. P. Abbott, R. Abbott, T. D. Abbott, F. Acernese, K. Ackley, C. Adams, T. Adams, P. Addesso, R. X. Adhikari, V. B. Adya et al., Multi-messenger Observations of a Binary Neutron Star Merger, Astrophys. J. Lett. 848, L12 (2017). 
[3] M. Shibata, S. Fujibayashi, K. Hotokezaka, K. Kiuchi, K. Kyutoku, Y. Sekiguchi, and M. Tanaka, Modeling GW170817 based on numerical relativity and its implications, Phys. Rev. D 96, 123012 (2017).

[4] A. Bauswein, O. Just, H. T. Janka, and N. Stergioulas, Neutronstar radius constraints from GW170817 and future detections, Astrophys. J. Lett. 850, L34 (2017).

[5] K. Kiuchi, K. Kyutoku, M. Shibata, and K. Taniguchi, Revisiting the lower bound on tidal deformability derived by AT 2017gfo, Astrophys. J. Lett. 876, L31 (2019).

[6] B. P. Abbott, R. Abbott, T. D. Abbott, F. Acernese, K. Ackley, C. Adams, T. Adams, P. Addesso, R. X. Adhikari, V. B. Adya et al. (LIGO Scientific and Virgo and Fermi-GBM and INTEGRAL Collaborations), Gravitational Waves and Gamma-rays from a Binary Neutron Star Merger: GW170817 and GRB 170817A, Astrophys. J. Lett. 848, L13 (2017).

[7] B. D. Metzger, T. A. Thompson, and E. Quataert, A magnetar origin for the kilonova ejecta in GW170817, Astrophys. J. Lett. 856, 101 (2018).

[8] S. Ai, H. Gao, Z. G. Dai, X. F. Wu, A. Li, B. Zhang, and M. Z. $\mathrm{Li}$, The allowed parameter space of a long-lived neutron star as the merger remnant of GW170817, Astrophys. J. Lett. 860, 57 (2018).

[9] S. Z. Li, L. D. Liu, Y. W. Yu, and B. Zhang, What Powered the Optical Transient AT2017gfo Associated with GW170817?, Astrophys. J. Lett. 861, L12 (2018).

[10] Y. W. Yu, L. D. Liu, and Z. G. Dai, A long-lived remnant neutron star after GW170817 inferred from its associated kilonova, Astrophys. J. Lett. 861, 114 (2018).

[11] B. Margalit and B. D. Metzger, Constraining the Maximum Mass of Neutron Stars From Multi-Messenger Observations of GW170817, Astrophys. J. Lett. 850, L19 (2017).

[12] L. Rezzolla, E. R. Most, and L. R. Weih, Using gravitationalwave observations and quasi-universal relations to constrain the maximum mass of neutron stars, Astrophys. J. Lett. 852, L25 (2018).

[13] M. Ruiz, S. L. Shapiro, and A. Tsokaros, GW170817, General Relativistic Magnetohydrodynamic Simulations, and the Neutron Star Maximum Mass, Phys. Rev. D 97, 021501(R) (2018).

[14] M. Shibata, E. Zhou, K. Kiuchi, and S. Fujibayashi, Constraint on the maximum mass of neutron stars using GW170817 event, Phys. Rev. D 100, 023015 (2019).

[15] B. P. Abbott, R. Abbott, T. D. Abbott, F. Acernese, K. Ackley, C. Adams, T. Adams, P. Addesso, R. X. Adhikari, V. B. Adya et al. (LIGO Scientific and Virgo Collaborations), Properties of the Binary Neutron Star Merger GW170817, Phys. Rev. X 9, 011001 (2019).

[16] B. P. Abbott, R. Abbott, T. D. Abbott, F. Acernese, K. Ackley, C. Adams, T. Adams, P. Addesso, R. X. Adhikari, V. B. Adya et al. (LIGO Scientific and Virgo Collaborations), GW170817: Measurements of Neutron Star Radii and Equation of State, Phys. Rev. Lett. 121, 061101 (2018).

[17] B. P. Abbott, R. Abbott, T. D. Abbott, F. Acernese, K. Ackley, C. Adams, T. Adams, P. Addesso, R. X. Adhikari, V. B. Adya et al. (LIGO Scientific and Virgo), Model comparison from LIGO-Virgo data on GW170817's binary components and consequences for the merger remnant, Classical Quantum Gravity 37, 045006 (2020).
[18] W. Kastaun and F. Ohme, Finite tidal effects in GW170817: observational evidence or model assumptions?, Phys. Rev. D 100, 103023 (2019).

[19] S. De, D. Finstad, J. M. Lattimer, D. A. Brown, E. Berger, and C. M. Biwer, Tidal Deformabilities and Radii of Neutron Stars from the Observation of GW170817, Phys. Rev. Lett. 121, 091102 (2018); 121, 259902(E) (2018)].

[20] C. D. Capano, I. Tews, S. M. Brown, B. Margalit, S. De, S. Kumar, D. A. Brown, B. Krishnan, and S. Reddy, GW170817: Stringent constraints on neutron-star radii from multimessenger observations and nuclear theory, Nat. Astron. 4, 625 (2020).

[21] F. J. Fattoyev, J. Piekarewicz, and C. J. Horowitz, Neutron Skins and Neutron Stars in the Multimessenger Era, Phys. Rev. Lett. 120, 172702 (2018).

[22] E. Annala, T. Gorda, A. Kurkela, and A. Vuorinen, Gravitational-Wave Constraints on the Neutron-Star-Matter Equation of State, Phys. Rev. Lett. 120, 172703 (2018).

[23] C. Raithel, F. Özel, and D. Psaltis, Tidal deformability from GW170817 as a direct probe of the neutron star radius, Astrophys. J. Lett. 857, L23 (2018).

[24] E. P. Zhou, X. Zhou, and A. Li, Constraints on interquark interaction parameters with GW170817 in a binary strange star scenario, Phys. Rev. D 97, 083015 (2018).

[25] V. Paschalidis, K. Yagi, D. Alvarez-Castillo, D. B. Blaschke, and A. Sedrakian, Implications from GW170817 and I-Love-Q relations for relativistic hybrid stars, Phys. Rev. D 97, 084038 (2018).

[26] R. Nandi and P. Char, Hybrid stars in the light of GW170817, Astrophys. J. Lett. 857, 12 (2018).

[27] D. Radice, A. Perego, F. Zappa, and S. Bernuzzi, GW170817: Joint Constraint on the Neutron Star Equation of State from Multimessenger Observations, Astrophys. J. Lett. 852, L29 (2018).

[28] D. Radice and L. Dai, Multimessenger Parameter Estimation of GW170817, Eur. Phys. J. A 55, 50 (2019).

[29] M. W. Coughlin, T. Dietrich, B. Margalit, and B. D. Metzger, Multimessenger Bayesian parameter inference of a binary neutron star merger, Mon. Not. R. Astron. Soc. 489, L91 (2019).

[30] B. P. Abbott, R. Abbott, T. D. Abbott, F. Acernese, K. Ackley, C. Adams, T. Adams, P. Addesso, R. X. Adhikari, V. B. Adya et al. (LIGO Scientific and Virgo Collaborations), GW190425: Observation of a Compact Binary Coalescence with Total Mass $\sim 3.4 M_{\odot}$, Astrophys. J. Lett. 892, L3 (2020).

[31] T. Dietrich, M. W. Coughlin, P. T. Pang, M. Bulla, J. Heinzel, L. Issa, I. Tews, and S. Antier, New Constraints on the Supranuclear Equation of State and the Hubble Constant from Nuclear Physics-Multi-Messenger Astronomy, arXiv:2002.11355 [astroph.HE].

[32] P. Landry, R. Essick, and K. Chatziioannou, Nonparametric constraints on neutron star matter with existing and upcoming gravitational wave and pulsar observations, Phys. Rev. D 101, 123007 (2020).

[33] E. E. Flanagan and T. Hinderer, Constraining neutron star tidal Love numbers with gravitational wave detectors, Phys. Rev. D 77, 021502(R) (2008).

[34] J. Vines, E. E. Flanagan, and T. Hinderer, Post-1-Newtonian tidal effects in the gravitational waveform from binary inspirals, Phys. Rev. D 83, 084051 (2011). 
[35] M. Favata, Systematic Parameter Errors in Inspiraling Neutron Star Binaries, Phys. Rev. Lett. 112, 101101 (2014).

[36] K. Yagi and N. Yunes, Love can be tough to measure, Phys. Rev. D 89, 021303(R) (2014).

[37] B. D. Lackey and L. Wade, Reconstructing the neutron-star equation of state with gravitational-wave detectors from a realistic population of inspiralling binary neutron stars, Phys. Rev. D 91, 043002 (2015).

[38] L. Wade, J. D. E. Creighton, E. Ochsner, B. D. Lackey, B. F. Farr, T. B. Littenberg, and V. Raymond, Systematic and statistical errors in a bayesian approach to the estimation of the neutron-star equation of state using advanced gravitational wave detectors, Phys. Rev. D 89, 103012 (2014).

[39] T. Damour, A. Nagar, and L. Villain, Measurability of the tidal polarizability of neutron stars in late-inspiral gravitational-wave signals, Phys. Rev. D 85, 123007 (2012).

[40] T. Hinderer, B. D. Lackey, R. N. Lang, and J. S. Read, Tidal deformability of neutron stars with realistic equations of state and their gravitational wave signatures in binary inspiral, Phys. Rev. D 81, 123016 (2010).

[41] T. Damour and A. Nagar, Effective One Body description of tidal effects in inspiralling compact binaries, Phys. Rev. D 81, 084016 (2010).

[42] D. Bini, T. Damour, and G. Faye, Effective action approach to higher-order relativistic tidal interactions in binary systems and their effective one body description, Phys. Rev. D 85, 124034 (2012).

[43] D. Bini and T. Damour, Gravitational self-force corrections to two-body tidal interactions and the effective one-body formalism, Phys. Rev. D 90, 124037 (2014).

[44] S. Bernuzzi, A. Nagar, T. Dietrich, and T. Damour, Modeling the Dynamics of Tidally Interacting Binary Neutron Stars up to the Merger, Phys. Rev. Lett. 114, 161103 (2015).

[45] T. Hinderer, A. Taracchini, F. Foucart, A. Buonanno, J. Steinhoff, M. Duez, L. E. Kidder, H. P. Pfeiffer, M. A. Scheel, B. Szilagyi et al., Effects of Neutron-Star Dynamic Tides on Gravitational Waveforms Within the Effective-One-Body Approach, Phys. Rev. Lett. 116, 181101 (2016).

[46] T. Dietrich, S. Bernuzzi, and W. Tichy, Closed-form tidal approximants for binary neutron star gravitational waveforms constructed from high-resolution numerical relativity simulations, Phys. Rev. D 96, 121501(R) (2017).

[47] T. Dietrich, A. Samajdar, S. Khan, N. K. Johnson-McDaniel, R. Dudi, and W. Tichy, Improving the NRTidal model for binary neutron star systems, Phys. Rev. D 100, 044003 (2019).

[48] K. Kawaguchi, K. Kiuchi, K. Kyutoku, Y. Sekiguchi, M. Shibata, and K. Taniguchi, Frequency-domain gravitational waveform models for inspiraling binary neutron stars, Phys. Rev. D 97, 044044 (2018).

[49] A. Buonanno, B. R. Iyer, E. Ochsner, Y. Pan, and B. S. Sathyaprakash, Comparison of post-Newtonian templates for compact binary inspiral signals in gravitational-wave detectors, Phys. Rev. D 80, 084043 (2009).

[50] L. Blanchet, Gravitational radiation from post-newtonian sources and inspiralling compact binaries, Living Rev. Rel. 17, 2 (2014)

[51] T. Narikawa, N. Uchikata, K. Kawaguchi, K. Kiuchi, K. Kyutoku, M. Shibata, and H. Tagoshi, Discrepancy in tidal deformability of GW170817 between the Advanced LIGO twin detectors, Phys. Rev. Res. 1, 033055 (2019).
[52] J. Skilling, Nested sampling for general Bayesian computation, Bayesian Anal. 1, 833 (2006).

[53] J. Veitch and A. Vecchio, Bayesian coherent analysis of inspiral gravitational wave signals with a detector network, Phys. Rev. D 81, 062003 (2010).

[54] J. Veitch, V. Raymond, B. Farr, W. Farr, P. Graff, S. Vitale, B. Aylott, K. Blackburn, N. Christensen, M. Coughlin, V. Raymond, B. Farr, W. Farr, P. Graff, S. Vitale, B. Aylott, K. Blackburn, N. Christensen, M. Coughlin et al., Parameter estimation for compact binaries with ground-based gravitationalwave observations using the LALInference software library, Phys. Rev. D 91, 042003 (2015).

[55] LIGO Scientific Collaboration, LIGO Algorithm LibraryLALSuite, Free Software (GPL), 2018; https://doi.org/10.7935/ GT1W-FZ16.

[56] S. Khan, S. Husa, M. Hannam, F. Ohme, M. Pürrer, X. J. Forteza, and A. Bohé, Frequency-domain gravitational waves from nonprecessing black-hole binaries. II. A phenomenological model for the advanced detector era, Phys. Rev. D 93 , 044007 (2016).

[57] A. Taracchini, A. Buonanno, Y. Pan, T. Hinderer, M. Boyle, D. A. Hemberger, L. E. Kidder, G. Lovelace, A. H. Mroue, H. P. Pfeiffer et al., Effective-one-body model for black-hole binaries with generic mass ratios and spins, Phys. Rev. D 89, 061502(R) (2014).

[58] M. Pürrer, Frequency domain reduced order model of alignedspin effective-one-body waveforms with generic mass-ratios and spins, Phys. Rev. D 93, 064041 (2016).

[59] A. Bohé, S. Marsat, and L. Blanchet, Next-to-next-to-leading order spin-orbit effects in the gravitational wave flux and orbital phasing of compact binaries, Classical Quantum Gravity 30, 135009 (2013).

[60] K. G. Arun, A. Buonanno, G. Faye, and E. Ochsner, Higherorder spin effects in the amplitude and phase of gravitational waveforms emitted by inspiraling compact binaries: Ready-touse gravitational waveforms, Phys. Rev. D 79, 104023 (2009); 84, 049901(E) (2011)].

[61] B. Mikoczi, M. Vasuth, and L. A. Gergely, Self-interaction spin effects in inspiralling compact binaries, Phys. Rev. D 71, 124043 (2005).

[62] T. Hinderer, Tidal Love numbers of neutron stars, Astrophys. J. Lett. 677, 1216 (2008).

[63] K. Kiuchi, K. Kawaguchi, K. Kyutoku, Y. Sekiguchi, M. Shibata, and K. Taniguchi, Sub-radian-accuracy gravitational waveforms of coalescing binary neutron stars in numerical relativity, Phys. Rev. D 96, 084060 (2017).

[64] J. Steinhoff, T. Hinderer, A. Buonanno, and A. Taracchini, Dynamical Tides in General Relativity: Effective Action and Effective-One-Body Hamiltonian, Phys. Rev. D 94, 104028 (2016).

[65] B. D. Lackey, M. Pürrer, A. Taracchini, and S. Marsat, Surrogate model for an aligned-spin effective one body waveform model of binary neutron star inspirals using Gaussian process regression, Phys. Rev. D 100, 024002 (2019).

[66] M. Soares-Santos, D. E. Holz, J. Annis, R. Chornock, K. Herner, E. Berger, D. Brout, H. Chen, R. Kessler, M. Sako et al. (DES and Dark Energy Camera GW-EM Collaborations), The Electromagnetic Counterpart of the Binary Neutron Star Merger LIGO/Virgo GW170817. I. Discovery of the Optical 
Counterpart Using the Dark Energy Camera, Astrophys. J. Lett. 848, L16 (2017).

[67] M. Burgay, N. D'Amico, A. Possenti, R. N. Manchester, A. G. Lyne, B. C. Joshi, M. A. McLaughlin, M. Kramer, J. M. Sarkissian, F. Camilo et al., An Increased estimate of the merger rate of double neutron stars from observations of a highly relativistic system, Nature (London) 426, 531 (2003).

[68] K. Stovall, P. C. C. Freire, S. Chatterjee, P. B. Demorest, D. R. Lorimer, M. A. McLaughlin, N. Pol, J. van Leeuwen, R. S. Wharton, B. Allen et al., PALFA Discovery of a Highly Relativistic Double Neutron Star Binary, Astrophys. J. Lett. 854, L22 (2018).

[69] P. Ajith, M. Hannam, S. Husa, Y. Chen, B. Brugmann, N. Dorband, D. Muller, F. Ohme, D. Pollney, C. Reisswig, L. Santamaria, and J. Seiler, Inspiral-Merger-Ringdown Waveforms for Black-Hole Binaries with Non-Precessing Spins, Phys. Rev. Lett. 106, 241101 (2011).

[70] E. Racine, Analysis of spin precession in binary black hole systems including quadrupole-monopole interaction, Phys. Rev. D 78, 044021 (2008).

[71] P. A. R. Ade, N. Aghanim, M. I. R. Alves, C. Armitage-Caplan, M. Arnaud, M. Ashdown, F. Atrio-Barandela, J. Aumont, H. Aussel, C. Baccigalupi et al. (Planck Collaboration), Planck 2013 results. I. Overview of products and scientific results, Astron. Astrophys. 571, A1 (2014).

[72] B. P. Abbott, R. Abbott, T. D. Abbott, F. Acernese, K. Ackley, C. Adams, T. Adams, P. Addesso, R. X. Adhikari, V. B. Adya et al. (LIGO Scientific and Virgo Collaborations), GWTC-1: A Gravitational-Wave Transient Catalog of Compact Binary Mergers Observed by LIGO and Virgo during the First and Second Observing Runs, Phys. Rev. X 9, 031040 (2019).

[73] I. Harry and T. Hinderer, Observing and measuring the neutronstar equation-of-state in spinning binary neutron star systems, Classical Quantum Gravity 35, 145010 (2018).

[74] A. Samajdar and T. Dietrich, Waveform systematics for binary neutron star gravitational wave signals: effects of the point- particle baseline and tidal descriptions, Phys. Rev. D 98, 124030 (2018).

[75] A. Samajdar and T. Dietrich, Waveform systematics for binary neutron star gravitational wave signals: Effects of spin, precession, and the observation of electromagnetic counterparts, Phys. Rev. D 100, 024046 (2019).

[76] F. Messina, R. Dudi, A. Nagar, and S. Bernuzzi, Quasi-5.5PN TaylorF2 approximant for compact binaries: point-mass phasing and impact on the tidal polarizability inference, Phys. Rev. D 99, 124051 (2019).

[77] H. Mueller and B. D. Serot, Relativistic mean field theory and the high density nuclear equation of state, Nucl. Phys. A 606, 508 (1996).

[78] B. D. Lackey, M. Nayyar, and B. J. Owen, Observational constraints on hyperons in neutron stars, Phys. Rev. D 73, 024021 (2006).

[79] H. Müther, M. Prakash, and T. Ainsworth, The nuclear symmetry energy in relativistic Brueckner-Hartree-Fock calculations, Phys. Lett. B 199, 469 (1987).

[80] A. Akmal, V. R. Pandharipande, and D. G. Ravenhall, The Equation of state of nucleon matter and neutron star structure, Phys. Rev. C 58, 1804 (1998).

[81] R. B. Wiringa, V. Fiks, and A. Fabrocini, Equation of state for dense nucleon matter, Phys. Rev. C 38, 1010 (1988).

[82] https://www.gw-openscience.org

[83] A. Bohe, L. Shao, A. Taracchini, A. Buonanno, S. Babak, I. W. Harry, I. Hinder, S. Ossokine, M. Pürrer, V. Raymond, L. Shao, A. Taracchini, A. Buonanno, S. Babak, I. W. Harry, I. Hinder, S. Ossokine, M. Pürrer, V. Raymond et al., Improved effectiveone-body model of spinning, nonprecessing binary black holes for the era of gravitational-wave astrophysics with advanced detectors, Phys. Rev. D 95, 044028 (2017).

[84] M. Pürrer, Frequency domain reduced order models for gravitational waves from aligned-spin compact binaries, Classical Quantum Gravity 31, 195010 (2014). 